\title{
EFICIÊNCIA DE PRODUÇÃO: UM ENFOQUE BAYESIANO
}

\author{
JULIANA GARCIA CESPEDES
}

\begin{abstract}
Dissertação apresentada à Escola Superior de Agricultura "Luiz de Queiroz", Universidade de São Paulo, para obtenção do título de Mestre em Agronomia, Área de Concentração: Estatística e Experimentação Agronômica.
\end{abstract}

P I R A C I C A B A

Estado de São Paulo - Brasil

Dezembro - 2003 


\title{
EFICIÊNCIA DE PRODUÇÃO: UM ENFOQUE BAYESIANO
}

\author{
JULIANA GARCIA CESPEDES
}

Licenciada em Matemática

Orientadora: $\operatorname{Prof}^{\underline{a}} \operatorname{Dr}^{\underline{a}}$ ROSELI APARECIDA LEANDRO

Dissertação apresentada à Escola Superior de Agricultura "Luiz de Queiroz", Universidade de São Paulo, para obtenção do título de Mestre em Agronomia, Área de Concentração: Estatística e Experimentação Agronômica.

P I R A C I C A B A

Estado de São Paulo - Brasil

Dezembro - 2003 
Dados Internacionais de Catalogação na Publicação (CIP) DIVISÃO DE BIBLIOTECA E DOCUMENTAÇÃO - ESALQ/USP

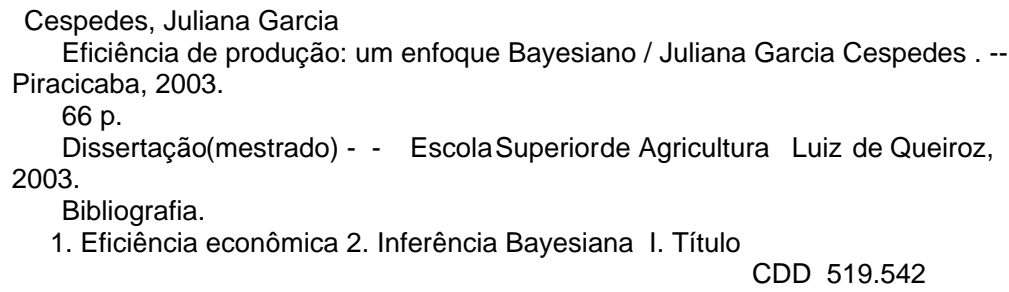

\section{"Permitida a cópia total ou parcial deste documento, desde que citada a fonte - $O$ autor"}




\section{DEDICATÓRIA}

A

\section{DEUS}

Senhor todo poderoso.

Aos meus pais,

João Garcia Cespedes e

Nair da Graças Lomazini Garcia,

fundamentais nesta conquista, pelo apoio, amor e con-

fiança, que nortearam minha vida e me fizeram progredir.

Ao meu irmão

\section{Luís Fernando Garcia Cespedes}

a confiança e o apoio. 


\section{AGRADECIMENTOS}

À Prof ${ }^{\underline{a}} \operatorname{Dr}^{\underline{a}}$ Roseli Aparecida Leandro a orientação, a amizade e apoio, que foram fundamentais para a realização deste trabalho.

Aos meus pais João Garcia Cespedes e Nair das Graças Lomazini Garcia e ao meu irmão Luís Fernando Garcia Cespedes a força, o amor incondicional, a confiança e a dedicação que me deram nos momentos decisivos.

Aos amigos do mestrado e doutorado, em especial ao Adriano Ferreti Borgatto, Antônio Willians Moita, Ana Alice Pilon, e aos amigos Tiago Badocha, Fernanda Hardt, Adriano Mirkeschkin, Rosemeire Batistela, Jucelene Gimenez, Fernando Damasceno o apoio e a compreensão.

Ao Prof $\underline{\underline{o}}$ Dro José Silvio Govone, o incentivo.

Aos professores e funcionários do Departamento de Ciências Exatas

da ESALQ/USP, em especial a $\operatorname{Prof}^{\underline{a}} \operatorname{Dr}^{\underline{a}}$ Clarice Garcia Borges Demétrio, que me propiciaram condições para a realização deste trabalho.

À CNPQ o apoio financeiro.

Aos pesquisadores Oscar Tupy e Geraldo da Silva e Souza da EMBRAPA que gentilmente cederam dados para a realização deste trabalho.

Ao pesquisador Mark Steel a ajuda científica.

Ao responsável pelos laboratórios de informática, Jorge Alexandre Wiendl, a ajuda.

À Santo Antônio e Nossa Senhora Aparecida as graças recebidas. 


\section{SUMÁRIO}

Página

LISTA DE FIGURAS . . . . . . . . . . . . . . . . . . . . vii

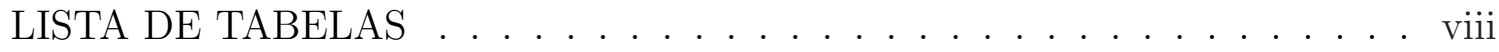

RESUMO ........................ . . . ix

SUMMARY ............................... xi

$1 \quad$ INTRODUÇÃ $\ldots \ldots \ldots \ldots \ldots$

2 REVISÃO DE LITERATURA . . . . . . . . . . . . 3

2.1 História da Estatística Bayesiana . . . . . . . . . . . . . . 3

$2.2 \quad$ O que é Inferência Bayesiana . . . . . . . . . . . . . . . . . . 4

2.3 Teorema de Bayes ......................... 4

2.4 Distribuição a priori . . . . . . . . . . . . . . . 5

2.4.1 Distribuições ajustadas a freqüências relativas . . . . . . . . . . . 7

2.4.2 Distribuições ajustadas a expectativas subjetivas . . . . . . . . . . . 7

2.4.3 Distribuiçõos a priori conjugadas . . . . . . . . . . . . . 8

2.4.4 Distribuições objetivas, distribuições não-informativas . . . . . . . . . 8

2.5 Verossimilhança ....................... 10

2.6 Distribuição a posteriori . . . . . . . . . . . . . . . . . 10

$2.7 \quad$ Intervalo de Credibilidade . . . . . . . . . . . . . . . . . . 11

2.8 Obtenção de resumos de interesse através de simulação . . . . . . . . . 11

2.8.1 Integração Monte Carlo . . . . . . . . . . . . . . . . . . . . . . . . . 12

2.8.2 Monte Carlo com cadeia de Markov . . . . . . . . . . . . . . . 13

2.8.3 Algoritmo Metropolis-Hastings . . . . . . . . . . . . . . . . . . 15 
2.8.4 Amostrador de Gibbs . . . . . . . . . . . . . . . . . . . 17

2.8.5 "Slice sampling" . . . . . . . . . . . . . . . . . . . . . . . . . . . . 18

2.9 Conceito de Firma . . . . . . . . . . . . . . . . . . . . . 21

2.10 Função de produção . . . . . . . . . . . . . . . . . . . . . . . 23

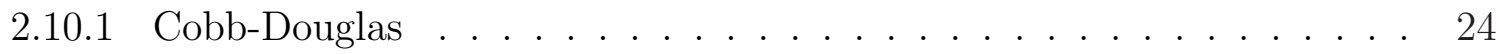

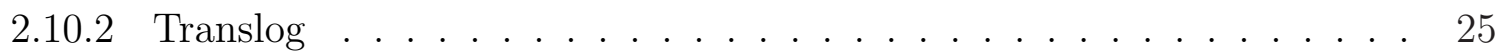

2.10 .3 Retornos à escala . . . . . . . . . . . . . . . . . . . 26

2.10.4 Produto Marginal . . . . . . . . . . . . . . . . . . . . 26

2.11 Fronteira de produção . . . . . . . . . . . . . . . . 27

2.11.1 Fronteira de produção determinística . . . . . . . . . . . . . . . 27

2.11.2 Fronteira de produção estocástica . . . . . . . . . . . . . . . . . . 28

2.12 Eficiência Produtiva . . . . . . . . . . . . . . . . . . 29

2.12.1 Eficiência técnica . . . . . . . . . . . . . . . . . 30

2.12.2 Modelando os efeitos de ineficiência . . . . . . . . . . . . . . 33

3 MATERIAL E MÉTODOS . . . . . . . . . . . . 37

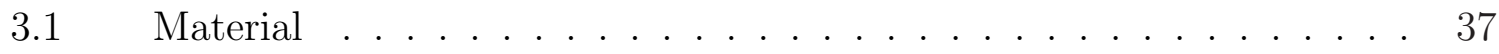

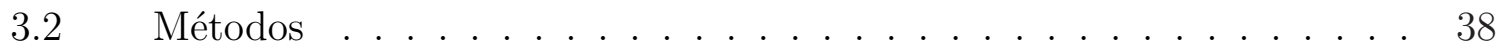

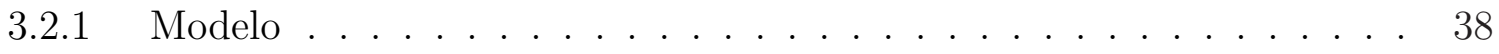

3.2.2 Distribuição a priori para os parâmetros . . . . . . . . . . . . . . 43

3.2.3 Distribuição conjunta a posteriori . . . . . . . . . . . . . . . . 45

3.2.4 Distribuições condicionais completas a posteriori . . . . . . . . . . . . 46

3.2.5 Simulação do conjunto de dados . . . . . . . . . . . . . . . . . . 48

3.2.6 Alguns comentários sobre a implementação do programa . . . . . . . . 49

4 RESULTADOS E DISCUSSÃO . . . . . . . . . . . 51

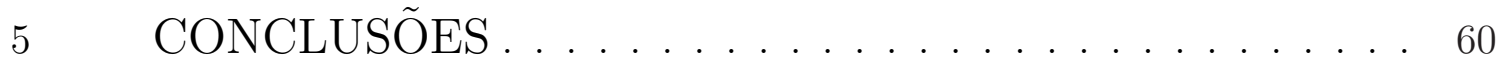

REFERÊNCIAS BIBLIOGRÁFICAS . . . . . . . . . . . . 61 


\section{LISTA DE FIGURAS}

Página

1 Intervalo de Credibilidade . . . . . . . . . . . . . . . . . 11

2 "Slice sampling" . . . . . . . . . . . . . . . . . . . . . . . . 19

3 Função de produção . . . . . . . . . . . . . . . . . . . . . 23

4 Forma funcional Cobb-Douglas . . . . . . . . . . . . . . . . . . . 24

5 Forma funcional Translog . . . . . . . . . . . . . . . . . . 25

6 Fronteira de Produção Determinística . . . . . . . . . . . . . . . . . . . . 28

7 Fronteira de Produção Estocástica . . . . . . . . . . . . . . . . . . . . 29

8 Eficiência . . . . . . . . . . . . . . . . . 30

9 Produtividade e Eficiência técnica . . . . . . . . . . . . . . . . 31

10 Fronteira de Produção . . . . . . . . . . . . . . . . . . . . 35

11 Traço e densidade da distribuição marginal a posteriori do parâmetro z na análise MHGS. . . . . . . . . . . . . . . . . . . . . . . 54

12 Autocorrelação da amostra gerada para o parâmetro $z=\left(z_{1}, \ldots, z_{5}\right)$ na análise MHGS. . . . . . . . . . . . . . . . . . . 55

13 Traço e densidade da distribuição marginal a posteriori do parâmetro z na análise SS. . . . . . . . . . . . . . . . . . 56

14 Autocorrelação da amostra gerada para o parâmetro $z=\left(z_{1}, \ldots, z_{5}\right)$ na análise SS. . . . . . . . . . . . . . . . . . . 57 


\section{LISTA DE TABELAS}

Página

1 Séries temporais . . . . . . . . . . . . . . . . . 31

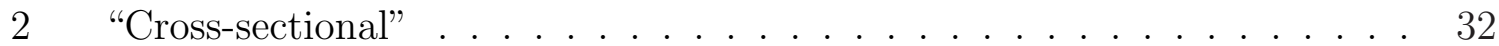

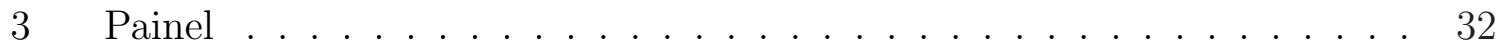

4 Resumo a posteriori dos parâmetros de interesse na análise MHGS. . . . 52

$5 \quad$ Resumo a posteriori dos parâmetros de interesse na análise SS. . . . . . . 53

6 Resumo a posteriori do vetor de ineficiências na análise MHGS. . . . . . 58

7 Resumo a posteriori do vetor de ineficiências na análise SS. . . . . . . . . 58

8 Índice de Eficiência Técnica . . . . . . . . . . . . . . . . . . . . . . 59 


\title{
EFICIÊNCIA DE PRODUÇÃO: UM ENFOQUE BAYESIANO
}

\author{
Autora: JULIANA GARCIA CESPEDES

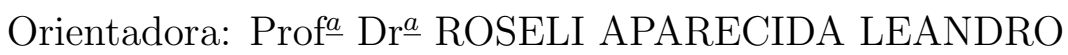

\section{RESUMO}

O uso de fronteira de produção estocástica com múltiplos produtos tem despertado um interesse especial em áreas da economia que defrontam-se com o problema de quantificar a eficiência técnica de firmas. Na estatística clássica, quando se defronta com firmas que possuem vários produtos, as funções custo ou demanda são mais utilizadas para calcular essa eficiência, mas isso requer uma quantidade maior de informações sobre os dados, além das quantidades de insumos e produtos, também são necessários seus preços e custos. Quando existem apenas informações sobre os insumos $(\boldsymbol{x})$ e os produtos $(\boldsymbol{y})$ há a necessidade de se trabalhar com a função de produção e a inexistência de estatísticas suficientes para alguns parâmetros tornam a análise díficil. A abordagem Bayesiana pode se tornar uma ferramenta muito útil para esse caso, pois é possível obter uma amostra da distribuição de probabilidade dos parâmetros do modelo, possibilitando a obtenção de resumos de interesse. Para obter 
as amostras dessas distribuições métodos Monte Carlo com cadeias de Markov, tais como, amostrador de Gibbs, Metropolis-Hastings e "Slice sampling" são utilizados. 


\title{
PRODUCTION EFFICIENCY: A BAYESIAN APPROACH
}

\author{
Author: JULIANA GARCIA CESPEDES \\ Adviser: $\operatorname{Prof}^{\underline{a}} \operatorname{Dr}^{\underline{a}} \underline{\text { ROSELI APARECIDA LEANDRO }}$
}

\section{SUMMARY}

The use of stochastic production frontier with multiple-outputs has been waking up a special interest in areas of the economy that are confronted with the problem of quantifying the technical efficiency of firms. In the classic statistics, when it is confronted with firms that possess several outputs, cost or profit functions are more used to calculate that efficiency, but that requests an amount larger of information about data set, besides the amounts of inputs and outputs, are also necessary your prices and costs. When just exist information on inputs $(\boldsymbol{x})$ and outputs $(\boldsymbol{y})$ there is need to work with the production function and the lack of enough statistics for some parameters turn the difficult analysis. Bayesian approach can become a useful tool for that case, because is possible to obtain a sample of the distribution of probability of the parameters of the model, making possible the obtaining of summaries of interest. To obtain samples of those distributions methods 
Markov chains Monte Carlo, that is, Gibbs sampling, Metropolis-Hastings and Slice sampling are used. 


\section{INTRODUÇÃO}

Em algumas áreas da economia há o interesse em quantificar a eficiência técnica de uma determinada firma. Por firma, entende-se lacticínio, cooperativa, fazenda, banco etc. Essa eficiência pode ser calculada de várias maneiras, através da função de produção ou das funções custo ou demanda. Quando se trabalha com um único produto, os cálculos são simples, independente da abordagem utilizada, mas face ao problema de calcular a eficiência de uma firma com múltiplos produtos, a inferência Bayesiana é de grande importância.

$\mathrm{Na}$ estatística clássica, quando se defronta com firmas que possuem vários produtos, as funções custo ou demanda são mais utilizadas para calcular essa eficiência, pelo motivo de facilidades matemáticas, mas isso requer uma quantidade maior de informações sobre os dados. Além das quantidades de insumos e produtos, também são necessários seus preços e custos. Quando existem apenas informações sobre os insumos $(\boldsymbol{x})$ e os produtos $(\boldsymbol{y})$ há a necessidade de se trabalhar com a função de produção.

A maioria das pesquisas em análises de função de produção com múltiplos produtos utilizam uma função de transformação, que descreve a tecnologia de produção através da relação:

$$
f(\boldsymbol{x}, \boldsymbol{y})=0
$$

sendo $\boldsymbol{y} \in \Re^{P}$ um vetor de $P$ produtos e $\boldsymbol{x} \in \Re^{S}$ um vetor de $S$ insumos.

Mesmo assumindo a separabilidade da função de transformação, ou seja, se for possível escrevê-la como:

$$
g(\boldsymbol{y})=h(\boldsymbol{x}),
$$


a estimação econométrica desse modelo é difícil por duas razões:

i) uma única função, como a função de transformação, não é suficiente para representar um modelo estatístico para $p>2$ variáveis. Geralmente, é necessário um sistema com $p$ equações, e ainda, apenas escrever um modelo de regressão para $p$ produtos ignoraria as restrições da produção em conjunto, e

ii) $g(\boldsymbol{y})$ depende de parâmetros desconhecidos, isto é, $g(\boldsymbol{y})$ não constitui uma estatística suficiente para estimar $h(\cdot)$.

Alguns trabalhos que se encontram na literatura que utilizam a abordagem econométrica são Adams et al. (1999) e Lothgren (1997), que assumem a função de transformação separável.

Existe uma extensa literatura (Seiford \& Thrall, 1990; Lovell, 1993; Ali \& Seiford, 1993; Lovell, 1994; Charnes et al., 1995; Seiford, 1996; Pereira Filho, 2000; Souza, 2003) que utiliza a abordagem não paramétrica (sem medida de erro nos dados), usando técnicas de programação linear (DEA - Data Envelopment Analysis), mas está em desvantagem com a abordagem paramétrica pois não se pode considerar toda a incerteza envolvida na avaliação da ineficiência (Medici \& Migon ${ }^{1}, 2001$ ).

Com a abordagem Bayesiana esses problemas estão resolvidos. Consegue-se assumir uma forma geral para $g(\mathbf{y})$ e assim calcular todas as propriedades das características de interesse, inclusive a eficiência da firma, através de métodos de simulação Monte Carlo com cadeias de Markov (MCMC), tais como, amostrador de Gibbs, Metropolis-Hastings e "Slice sampling", superando as dificuldades estatísticas envolvidas na estimação clássica.

\footnotetext{
${ }^{1}$ MEDICI, E.V.; MIGON, H.S. Hierarchical Bayesian models applied to stochasic production frontier. migon@im.ufrj.br (10 Março 2003)
} 


\section{REVISÃO DE LITERATURA}

\subsection{História da Estatística Bayesiana}

Enquanto a probabilidade tem sido o objeto de estudo de centenas de anos, a estatística é um campo relativamente jovem (Carlin \& Louis, 1996). O campo não aflorou até os anos de 1920 e 30, quando R. A. Fisher desenvolveu a noção de verossimilhança para uma estimação geral, e Jerzy Neyman e Egon Pearson construíram a base para o teste de hipóteses clássico. Um tanto paradoxalmente, uma grande quantidade de atividades de pesquisas foi encorajada pela Segunda Guerra Mundial, que contou com uma nascente de problemas de difícil solução, e assim fundaram-se nos Estados Unidos e na Grã Bretanha as primeiras academias compostas por diversos pesquisadores motivados pela necessidade de solucioná-los. Por contraste, os métodos Bayesianos são muito mais velhos, datados de 1763, do artigo do Rev. Thomas Bayes: "An essay towards solving a problem in doctrine of chances" (Bayes, 1763).

A área Bayesiana despertava algum interesse em Laplace, Gauss e outros no século 19, mas um tanto ironicamente, foi grandemente ignorada pelos estatísticos do século 20. Felizmente, vários pesquisadores proeminentes, dentre os mais célebres Harold Jeffreys (um físico) e Arthur Bowley (um econometrista), continuaram a discutir as idéias Bayesianas (que chamavam de probabilidade inversa) durante esse período. Então, em meados de 1950, pesquisadores estatísticos tais como L.J. Savage, Bruno de Finetti, Dennis Lindley, Jack Kiefer e muitos outros começaram a defender métodos Bayesianos para superar certas deficiências do sis- 
tema clássico.

\subsection{O que é Inferência Bayesiana}

A inferência Bayesiana é o processo de encontrar um modelo de probabilidade para um conjunto de dados e resumir o resultado por uma distribuição de probabilidade sobre os parâmetros do modelo e sobre quantidades não observadas tais como predição para novas observações (Gelman et al., 2003).

A abordagem Bayesiana requer um modelo amostral (a verossimilhança) e, em adição, uma distribuição a priori para os parâmetros (Carlin \& Louis, 1996). Parâmetros desconhecidos são considerados aleatórios e todas as conclusões estão baseadas na distribuição condicional dos parâmetros sobre os dados observados, a distribuição a posteriori. A estatística Bayesiana está fundamentada no teorema de Bayes.

\subsection{Teorema de Bayes}

Suponha que $\quad \boldsymbol{y}^{\prime}=\left(y_{1}, \ldots, y_{n}\right) \quad$ é um vetor de $n$ observações cuja

distribuição de probabilidade $f(\boldsymbol{y} \mid \boldsymbol{\theta})$ depende dos valores de $k$ parâmetros $\boldsymbol{\theta}^{\prime}=\left(\theta_{1}, \ldots, \theta_{k}\right)$.

Para fazer a inferência sobre $\boldsymbol{\theta}$ dado $\boldsymbol{y}$, é necessário um modelo vindo de uma distribuição de probabilidade conjunta para $\boldsymbol{\theta}$ e $\boldsymbol{y}$ (Gelman et al., 2003). A função de densidade de probabilidade conjunta pode ser escrita como um produto de duas densidades que são chamadas de distribuição a priori $\pi(\boldsymbol{\theta})$ e distribuição amostral $f(\boldsymbol{y} \mid \boldsymbol{\theta})$ :

$$
f(\boldsymbol{\theta}, \boldsymbol{y})=\pi(\boldsymbol{\theta}) f(\boldsymbol{y} \mid \boldsymbol{\theta})
$$

A simples condição sobre os valores conhecidos dos dados $\boldsymbol{y}$, usando a 
propriedade de probabilidade condicional conhecida como teorema de Bayes, produz a densidade a posteriori:

$$
\pi(\boldsymbol{\theta} \mid \boldsymbol{y})=\frac{f(\boldsymbol{\theta}, \boldsymbol{y})}{f(\boldsymbol{y})}=\frac{\pi(\boldsymbol{\theta}) f(\boldsymbol{y} \mid \boldsymbol{\theta})}{f(\boldsymbol{y})},
$$

sendo $f(\boldsymbol{y})=\sum_{\boldsymbol{\theta}} \pi(\boldsymbol{\theta}) f(\boldsymbol{y} \mid \boldsymbol{\theta})$ a soma de todos os possíveis valores de $\boldsymbol{\theta}$, no caso em que $\boldsymbol{\theta}$ é discreto e $f(\boldsymbol{y})=\int_{\boldsymbol{\theta}} \pi(\boldsymbol{\theta}) f(\boldsymbol{y} \mid \boldsymbol{\theta})$ a integral de todos os possíveis valores de $\boldsymbol{\theta}$, no caso em que $\boldsymbol{\theta}$ é contínuo.

Uma forma equivalente de (1) omite o valor $f(\boldsymbol{y})$, que não depende de $\boldsymbol{\theta}$ e, com $\boldsymbol{y}$ fixo, pode então ser considerado constante, produzindo uma densidade a posteriori não normalizada:

$$
\pi(\boldsymbol{\theta} \mid \boldsymbol{y}) \propto \pi(\boldsymbol{\theta}) f(\boldsymbol{y} \mid \boldsymbol{\theta})
$$

Essa simples expressão contém o princípio técnico da inferência Bayesiana, em outras palavras, o teorema de Bayes conta que a distribuição para $\boldsymbol{\theta}$ posterior aos dados é proporcional ao produto da distribuição a priori para $\boldsymbol{\theta}$ e a verossimilhança de $\boldsymbol{\theta}$ dado $\boldsymbol{y}$ (Box \& Tiao, 1992). Pode-se justificar a presença do símbolo de proporcionalidade da seguinte maneira: quando se multiplica a função de verossimilhança por uma constante não se altera a inferência relativa ao parâmetro $\boldsymbol{\theta}$ e, assim, a distribuição a posteriori não será alterada (Leandro, 2001). A primeira tarefa de qualquer aplicação específica é desenvolver o modelo $f(\boldsymbol{\theta}, \boldsymbol{y})$ e executar os cálculos necessários para resumir $\pi(\boldsymbol{\theta} \mid \boldsymbol{y})$ de modo apropriado.

\subsection{Distribuição a priori}

Uma distribuição a priori (Box \& Tiao, 1992) que é usada para representar o que é conhecido sobre parâmetros desconhecidos antes de avaliar os dados, tem um importante papel na análise Bayesiana. Tal distribuição pode ser usada 
para representar o conhecimento a priori ou ignorância relativa. Em problemas de inferência científica, é possível que os dados "falem por si só", consequentemente, é apropriado conduzir a análise como se o estado de relativa ignorância existisse a priori. A compreensível intranqüilidade de alguns estatísticos sobre o uso da distribuição a priori está associada com o medo de que a priori pode dominar e distorcer "o que os dados estão tentando dizer". A análise Bayesiana pode produzir o contrário do que é temido, pode permitir aos dados questionar aspectos de um modelo de uma maneira que não seria possível em outros casos.

Uma propriedade básica da função de densidade de probabilidade $f(x)$ é que sua integral, ou soma no caso discreto, resultam em 1:

$$
\begin{aligned}
\int_{-\infty}^{\infty} f(x) d x & =1 \quad \text { caso contínuo } \\
\sum_{i=0}^{\infty} f\left(x_{i}\right) & =1 \quad \text { caso discreto. }
\end{aligned}
$$

Agora, se $f(x)$ é uniforme sobre toda a reta,

$$
f(x)=k, \quad k>0,
$$

ela não é uma densidade própria desde que a integral:

$$
\int_{-\infty}^{\infty} f(x) d x=k \int_{-\infty}^{\infty} d x,
$$

não existe independentemente de quão pequeno seja o valor de $k$ (Box \& Tiao, 1992). As funções de densidade desse tipo são chamadas de distribuições impróprias. Essas funçôes são frequentemente empregadas para representar um comportamento local da distribuição a priori na região onde a verossimilhança é apreciável, mas não sobre toda sua possivel variação. 


\title{
2.4.1 Distribuições ajustadas a freqüências relativas
}

\begin{abstract}
Em algumas aplicações existe uma quantidade significativa de evidências objetivas na qual pode-se basear uma distribuição a priori de um parâmetro $\theta$. Especificamente, experiências extensivas podem estar disponíveis e assim ter uma distribuição de freqüências sólida sobre os valores assumidos por $\boldsymbol{\theta}$ no passado. Neste caso, seria fácil selecionar um elemento da família de conjugadas naturais (Box \& Tiao, 1992) e assim, a única questão pendente para uma decisão subjetiva seria se esta distribuição se ajusta suficientemente bem para garantir seu uso como uma aproximação conveniente.
\end{abstract}

\subsubsection{Distribuições ajustadas a expectativas subjetivas}

O problema de ajustar uma distribuição a priori é muito mais difícil e interessante em situações em que não existe nenhuma base teórica objetiva para o ajuste. Nestas situações a distribuição a priori representa simplesmente a expectativa subjetiva do pesquisador. Desta forma o pesquisador transmite o seu "sentimento" à distribuição a priori, e portanto, o ajuste será feito através dessa informação. Neste momento, deverá haver a interação pesquisador-estatístico. Cabe ao estatístico, esclarecer a implicação de tal escolha, e, também, a responsabilidade de sugerir ao pesquisador medidas que resumam a sua subjetividade com a finalidade de verificar se o ajuste está seguindo uma linha correta. No entanto, mesmo estando o pesquisador satisfeito com a distribuição a priori adotada, via sua expectativa subjetiva, pode ocorrer que ele fique insatisfeito com algumas de suas implicações quando as vê refletidas na distribuição a posteriori, calculada depois de uma amostra tomada no presente. Neste caso, novamente, a interação pesquisador-estatístico é de suma importância. 


\subsubsection{Distribuições a priori conjugadas}

Uma família de distribuições a priori é conjugada se as distribuições a posteriori pertencerem à mesma família de distribuições.

Sejam $\boldsymbol{y}^{\prime}=\left(y_{1}, \ldots, y_{n}\right)$ observações independentes, identicamente distribuídas na família exponencial. Tem-se que a função densidade de probabilidade de $Y_{i}$ pode ser expressa como:

$$
f\left(y_{i} ; \boldsymbol{\theta}\right)=\exp \left\{a(\boldsymbol{\theta}) b\left(y_{i}\right)+c(\boldsymbol{\theta})+d\left(y_{i}\right)\right\}
$$

e sua função de verossimilhança por:

$$
L(\boldsymbol{\theta} \mid y) \propto \exp \left\{a(\boldsymbol{\theta}) \sum_{i=1}^{n} b\left(y_{i}\right)+n c(\boldsymbol{\theta})\right\} .
$$

Supondo a priori conjugada para $\boldsymbol{\theta}$ dada por:

$$
\pi\left(\boldsymbol{\theta} ; k_{1} ; k_{2}\right) \propto \exp \left\{k_{1} a(\boldsymbol{\theta})+k_{2} c(\boldsymbol{\theta})\right\}
$$

obtém-se a seguinte distribuição a posteriori:

$$
\pi(\boldsymbol{\theta} \mid y) \propto \exp \left\{a(\boldsymbol{\theta})\left[\sum_{i=1}^{n} b\left(y_{i}\right)+k_{1}\right]+c(\boldsymbol{\theta})\left[n+k_{2}\right]\right\} .
$$

\subsubsection{Distribuições objetivas, distribuições não-informativas}

Para julgar os dados em relação a uma priori de referência "neutra", o pesquisador utiliza o que comumente é chamado "princípio do júri". Os casos são tratados em um tribunal diante de um júri o qual é cuidadosamente escolhido de tal forma que não possua vínculo ou conexão com os principais interessados nem com os eventos do caso em questão (Leandro, 2001). A intenção é garantir claramente que a informação extraída dos dados ou depoimentos possa ser assumida como idéias que dominarão a informação a priori para que os membros do júri possam chegar a um veredicto final. 
Priori de referência é uma distribuição conveniente para ser utilizada como padrão a priori. A princípio pode ser dominada, ou não, pela verossimilhança ou informação presente fornecida pelos dados ou experimento, porém, na literatura em geral (Box \& Tiao, 1992) as prioris são dominadas por essas informações presentes, ou seja, pelos dados representados através da função de verossimilhança. Em algumas situações, o pesquisador sentirá que a informação disponível para avaliar a distribuição a priori não existe, ou ainda, quando ele deseja expressar, através da distribuição a priori, a sua indiferença ou ignorância com relação ao parâmetro $\theta$, existe a necessidade de explicitar, definir regras para selecionar, construir distribuições a priori para representar esse "conhecimento pobre" ou ignorância. Existe uma grande discussão referente a esse estado de espírito. É importante ressaltar que nunca se pode estar em completo estado de ignorância. O problema é como expressar a idéia do pouco conhecimento a respeito do parâmetro a priori em relação ao que os dados têm a contar?

Quando o valor de um parâmetro é completamente desconhecido, Jeffreys (1961), propôs duas regras para a escolha da distribuição a priori, as quais, segundo ele, atingem os casos mais comuns. Ele estabeleceu que:

1. se o parâmetro pode assumir qualquer valor em um domínio finito ou $(-\infty, \infty)$, uma distribuição a priori deveria ser considerada como uma distribuição uniforme. No caso de o parâmetro pertencer ao intervalo $(-\infty, \infty)$ essa distribuição não seria própria no sentido que sua integral no domínio não seria um.

2. no caso em que o parâmetro só pode assumir valores positivos, isto é, no intervalo $(0, \infty)$, então, a distribuição a priori do seu logaritmo deveria ser considerada como uniforme também em $(-\infty, \infty)$.

Tem-se que (Box \& Tiao, 1992):

Regra de Jeffreys para o caso uniparamétrico: A distribuição a priori 
para um único parâmetro $\theta$ é aproximadamente não informativa se for proporcional à raiz quadrada da medida de informação de Fisher.

Regra de Jeffreys para o caso multiparamétrico: A distribuição a priori para um conjunto de parâmetros é proporcional à raiz quadrada do determinante da matriz de informação de Fisher.

\subsection{Verossimilhança}

Usar o teorema de Bayes com um determinado modelo de probabilidade significa que os dados $\boldsymbol{y}$ afetam a distribuição a posteriori (2) somente através da função $f(\boldsymbol{y} \mid \boldsymbol{\theta})$, que, quando considerada como função de $\boldsymbol{\theta}$, para $\boldsymbol{y}$ fixo, é chamada de função de verossimilhança.

É através desta função que o conhecimento a priori sobre $\boldsymbol{\theta}$ é modificado. Esta função pode ser vista como a representação do que os dados têm a contar a respeito do parâmetro $\boldsymbol{\theta}$.

\subsection{Distribuição a posteriori}

Dada a distribuição a priori, o modelo de probabilidade $f(\boldsymbol{y} \mid \boldsymbol{\theta})$ e os dados $\boldsymbol{y}$, é possível calcular a distribuição de probabilidade $\pi(\boldsymbol{\theta} \mid \boldsymbol{y})$, a qual é chamada de distribuição a posteriori de $\boldsymbol{\theta}$.

A distribuição a posteriori $\pi(\boldsymbol{\theta} \mid \boldsymbol{y})$ contém toda a informação sobre o parâmetro $\boldsymbol{\theta}$. O gráfico da distribuição a posteriori é a melhor descrição do processo inferencial, mas nem sempre é possível esboçar esse gráfico, principalmente quando $\boldsymbol{\theta}$ é multiparamétrico. Assim, é conveniente apresentar alguns valores numéricos que possam resumir a informação contida na distribuição, tais como: média, mediana e intervalo de credibilidade dos parâmetros de interesse. 


\subsection{Intervalo de Credibilidade}

Intervalo de credibilidade (Figura 1), ou H.P.D., é o intervalo em que a densidade para todo ponto pertencente ao intervalo é maior do que para todo ponto não pertencente a ele, considerando o menor intervalo possível (Box \& Tiao, 1992). Para distribuições a posteriori simétricas, como a normal, tal intervalo é obtido de forma que seus extremos tenham densidades iguais. Esses intervalos são geralmente associados com 90, 95 ou 99\% da probabilidade total, sendo $95 \%$ o mais utilizado.

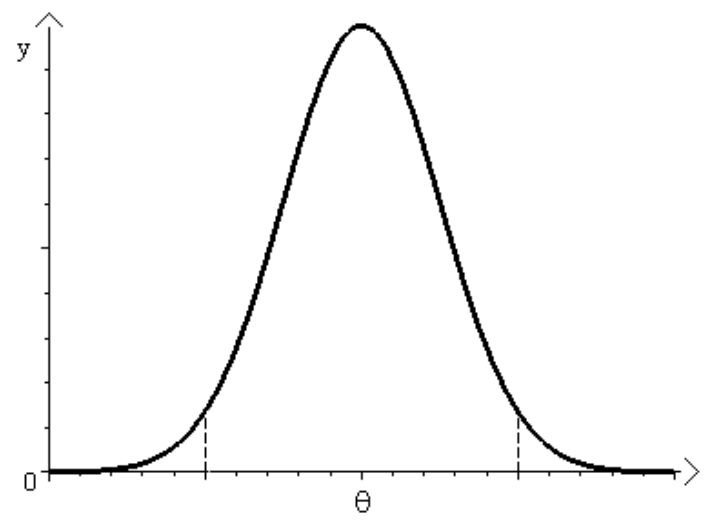

Figura 1 - Intervalo de Credibilidade

\subsection{Obtenção de resumos de interesse através de simulação}

Um dos tópicos mais ativos em Estatística Computacional é a inferência através de simulação iterativa, utilizando, especialmente o amostrador de Gibbs, o algoritmo Metropolis-Hastings e o "Slice sampling".

A idéia essencial da simulação iterativa é gerar valores de uma variável aleatória $\theta$ utilizando uma sequência de distribuições que converge iterativamente para a distribuição de origem ("target distribution") de $\theta$. A simulação iterativa é 
menos eficiente do que a simulação direta, que é simplesmente gerar amostras da distribuição fonte (original). No entanto, a simulação iterativa é aplicável em uma classe muito mais ampla de casos e desempenha um papel extremamente marcante em inferência Bayesiana no momento de calcular resumos de distribuições a posteriori extremamente complicadas.

\subsubsection{Integração Monte Carlo}

Métodos de simulação Monte Carlo são utilizados para calcular quantidades de interesse que são difíceis ou impossíveis de serem calculadas analiticamente. Um estudo de simulação é feito gerando milhares de observações de uma estrutura de interesse e estatísticas baseadas nesses valores simulados podem ser calculadas.

Suponha que $X$ é uma variável aleatória com densidade $f(x)$. Sabe-se que:

$$
\theta=E[g(X)]=\int g(x) f(x) d x
$$

A expressão (3) pode ser muito difícil, ou até impossível de ser calculada algebricamente, mas gerar elementos da distribuição com densidade $f(x)$ pode ser muito mais fácil.

Se as observações $X_{1}, \ldots, X_{n}$ são independentes e identicamente distribuídas com densidade $f(x)$, um estimador de (3) é:

$$
\hat{\theta}=\frac{1}{n} \sum_{j=1}^{n} g\left(X_{j}\right),
$$

que converge para $E[g(X)]$ com probabilidade 1 quando $n \rightarrow \infty$, pela Lei dos Grandes Números (Carlin \& Louis, 1996).

Sabe-se que $\hat{\theta}$ é somente a média amostral de observações independentes e temos que $\operatorname{Var}(\hat{\theta})=\frac{\operatorname{Var}[g(X)]}{n}$. Mas $\operatorname{Var}[g(X)]$ pode ser estimada pela variância amostral de $g\left(X_{i}\right)$, e assim a imprecisão (erro padrão estimado) para $\hat{\theta}$ é 
dado por:

$$
\widehat{s e}(\hat{\theta})=\sqrt{\frac{1}{n(n-1)} \sum_{j=1}^{n}\left[g\left(X_{i}\right)-\hat{\theta}\right]^{2}}
$$

Uma extensão da integração Monte Carlo é dada pela importance sampling (Leandro, 2001). Esse método é útil quando não se consegue gerar valores da densidade $f(x)$ diretamente. Nesse caso utiliza-se uma distribuição $h(x)$ da qual seja fácil amostrar:

$$
\theta=E[g(X)]=\int g(x) f(x) d x=\int g(x) \frac{f(x)}{h(x)} h(x) d x,
$$

e estimar $\theta$ :

$$
\hat{\theta} \approx \frac{1}{n} \sum_{j=1}^{n} \frac{g\left(X_{i}\right) f\left(X_{i}\right)}{h\left(X_{i}\right)}
$$

Em particular, pode-se considerar nas equações (3) e (4) que $f(x)=\pi(\theta \mid \boldsymbol{y})$ e desta forma obtém-se valores simulados para $\theta$ em que $\theta \in \Re$.

\subsubsection{Monte Carlo com cadeia de Markov}

Em geral, amostrar $X_{t}$, independentemente, da distribuição de $\pi(\cdot)$ não é factível, visto que $\pi(\cdot)$ pode ser muito diferente das distribuições padrões, tais como: normal, beta, gama etc.

Entretanto $\left\{X_{t}\right\}$ não precisa ser necessariamente independente. As $\left\{X_{t}\right\}$ podem ser geradas por qualquer processo que gera amostras através do suporte de $\pi(\cdot)$ numa proporção correta. Uma forma para construir essa amostra é através de uma cadeia de Markov que possua $\pi(\cdot)$ como distribuição estacionária (invariante), ou seja, que possua $\pi(\cdot)$ como distribuição de equilíbrio. Nem sempre tal distribuição existe.

Dependência de Markov é um conceito atribuído a um matemático russo Andrei Andreivich Markov que no ínicio do século 20 pesquisou a alternância 
de vogais e consoantes no poema "Onegim" de Poeshkin. Ele desenvolveu um modelo probabilístico no qual resultados sucessivos dependiam dos resultados de seus predecessores somente através do predecessor imediato.

$$
X^{(0)} X^{(1)} X^{(2)} \ldots X^{(n-2)} \underbrace{X^{(n-1)}} \overbrace{X^{(n)}} .
$$

Esse modelo permitiu, a ele, obter boas estimativas da freqüência de vogais no poema. Quase simultaneamente, o matemático francês Henri Poincaré estudou sequências de variáveis aleatórias que eram de fato cadeias de Markov.

Uma cadeia de Markov é um tipo especial de processo estocástico que cuida da caracterização de sequência de variáveis aleatórias. De especial interesse é a dinâmica e o comportamento limite da sequência.

Um processo estocástico pode ser definido como uma coleção de quantidades aleatórias $\left\{X^{(t)} \in S \mid t \in T\right\}$ para algum $T$. O conjunto $\left\{X^{(t)} \in S \mid t \in T\right\}$ é definido como um processo estocástico com espaço de estados $S$ e conjunto de índices T. Simplificando, uma cadeia de Markov é um processo estocástico, em que, dado o estado presente, passado e futuro são independentes. Essa propriedade pode ser formalizada através de:

$\operatorname{Pr}\left[X^{(n+1)} \in A \mid X^{(n)}=x, X^{(n-1)} \in A_{n-1}, \ldots X^{(0)} \in A_{0}\right]=\operatorname{Pr}\left[X^{(n+1)} \in A \mid X^{(n)}=x\right]$ para quaisquer conjuntos $A_{0}, \ldots, A_{n-1}, A \subset S$ e $x \in S$ (Gamerman, 1997).

Suponha a sequência de variáveis aleatórias $\left\{X_{0}, X_{1}, X_{2}, \ldots\right\}$ tal que em cada tempo $t \geq 0$ o próximo estado $X_{t+1}$ é amostrado da distribuição $P\left(X_{t+1} \mid X_{t}\right)$ a qual depende somente do estado atual da cadeia $X_{t}$, isto é, dado $X_{t}$, o próximo estado $X_{t+1}$ não depende mais da história da cadeia $\left\{X_{0}, X_{1}, X_{2}, \ldots, X_{t-1}\right\}$. Esta cadeia é chamada de cadeia de Markov e $P(\cdot \mid \cdot)$, é chamado de "kernel" de transição da cadeia e não depende de $t$ se a cadeia for homogênea no tempo.

Sujeito a condições de regularidade a cadeia irá gradualmente esquecendo seu estado inicial $P^{(t)}\left(\cdot \mid X_{0}\right)$, sendo que $P^{(t)}\left(X_{t} \mid X_{0}\right)$ é a distribuição de $X_{t}$ dado $X_{0}$, e convergirá para uma única distribuição estacionária ou invariante, que 
não depende de $t$ ou $X_{0}$. Esta distribuição estacionária será denotada por $\phi(\cdot)$. Com o crescimento de $t$ os pontos amostrados $\left\{X_{t}\right\}$ se parecerão mais e mais com amostras dependentes de $\phi(\cdot)$. Assim, depois de se desprezarem $m$ iterações ("burn-in"), os pontos $\left\{X_{t}, \quad t=m+1, \ldots, n\right\}$ serão aproximadamente amostras dependentes de $\phi(\cdot)$. Pode-se agora utilizar esta amostra para estimar a esperança $E[g(X)]$ em que $X$ tem distribuição $\phi(\cdot)$. Descartando o "burn-in" para os cálculos do estimador tem-se:

$$
\bar{g}=\frac{1}{n-m} \sum_{t=m+1}^{n} g\left(X_{t}\right),
$$

que é chamada de média ergódica.

A equação (5) mostra como uma cadeia de Markov pode ser utilizada para estimar $E[g(X)]$, em que a esperança é tomada sobre a distribuição estacionária $\phi(\cdot)$.

Esta parece fornecer uma solução para o problema, mas primeiro é necessário descobrir como construir cadeias de Markov tal que a sua distribuição estacionária $\phi(\cdot)$ seja precisamente a distribuição de interesse $f(x)$ que no caso Bayesiano é a distribuição conjunta a posteriori $\pi(\theta \mid \boldsymbol{y})$, isto é, $f(x)=\pi(\theta \mid \boldsymbol{y})$.

Apresentam-se a seguir três algoritmos: Metropolis-Hastings, amostrador de Gibbs e "Slice sampling", que permitem gerar cadeias de Markov cuja distribuição de equilíbrio seja precisamente $\pi(\theta \mid \boldsymbol{y})$.

\subsubsection{Algoritmo Metropolis-Hastings}

O algoritmo Metropolis-Hasting permite gerar uma amostra da distribuição conjunta a posteriori $\pi\left(\theta_{1}, \theta_{2}, \ldots, \theta_{k} \mid \boldsymbol{y}\right)$, a partir das distribuições condicionais completas, que podem possuir forma fechada ou não (Metropolis et al., 1953; Hastings, 1970; Chib \& Greenberg, 1995).

Os fatos principais desse método para gerar amostras de uma distribuição com densidade $\pi(\theta \mid \boldsymbol{y})=p(\theta)$ são: 
i) os cálculos dependem de $p(\theta)$ somente através de razões da forma $\frac{p\left(\theta^{\prime}\right)}{p(\theta)}$ em que $\theta$ e $\theta^{\prime}$ são pontos amostrais. Assim, a constante normalizadora não precisa ser conhecida pois será cancelada no quociente;

ii) nenhuma fatoração de $p(\theta)$ será necessária;

iii) os métodos são facilmente implementados, e

iv) a sequência de amostras é obtida através de uma cadeia de Markov.

Seja a cadeia de Markov $\left\{\theta_{0}, \theta_{1}, \theta_{2}, \ldots, \theta_{z}, \ldots\right\}$. Para o algoritmo de Metropolis-Hastings, a cada tempo $t \geq 0$ o próximo estado $\theta_{t+1}$ é escolhido, primeiramente, amostrando um ponto candidato $Y$ da distribuição proposta $q\left(\cdot \mid \theta_{t}\right)$ (a distribuição proposta pode depender do ponto atual, $\left.\theta_{t}\right)$.

O ponto candidato $Y$ é então aceito com probabilidade $\alpha\left(\theta_{t}, Y\right)$ sendo que

$$
\alpha\left(\theta_{t}, Y\right)=\min \left\{1, \frac{p(Y) q\left(\theta_{t} \mid Y\right)}{p\left(\theta_{t}\right) q\left(Y \mid \theta_{t}\right)}\right\} .
$$

Quando $q(\cdot \mid \cdot)$ for simétrica

$$
\alpha\left(\theta_{t}, Y\right)=\min \left\{1, \frac{p(Y)}{p\left(\theta_{t}\right)}\right\} .
$$

Se o ponto candidato $Y$ for aceito, o próximo estado será $\theta_{t+1}=Y$. Se o candidato for rejeitado, a cadeia não se moverá, isto é, $\theta_{t+1}=\theta_{t}$.

O algoritmo Metropolis-Hastings (Gilks et al., 1997) é dado por:

Inicie $\theta_{0}$;

Faça $t=0$

Repita \{

Amostre um ponto $Y$ de $q\left(\cdot \mid \theta_{t}\right)$

Amostre um valor $U$ de uma uniforme $(0,1)$

Se $U \leq \alpha\left(\theta_{t}, Y\right)$ faça $\theta_{t+1}=Y$

caso contrário faça $\theta_{t+1}=\theta_{t}$ 
Incremente $t$

em que $\alpha\left(\theta_{t}, Y\right)=\min \left\{1, \frac{p(Y) q\left(\theta_{t} \mid Y\right)}{p\left(\theta_{t}\right) q\left(Y \mid \theta_{t}\right)}\right\}$.

\subsubsection{Amostrador de Gibbs}

O amostrador de Gibbs é um caso especial do Metropolis-Hastings, que permite gerar uma amostra da distribuição conjunta a posteriori $\pi\left(\theta_{1}, \theta_{2}, \ldots, \theta_{k} \mid \boldsymbol{y}\right)$, desde que as distribuições condicionais cheias possuam forma fechada, no sentido que seja fácil amostrar de seus elementos (Gelfand et al., 1990; Casella \& George, 1992; Gelfand, 2000).

Suponha que $\boldsymbol{\theta}$, um vetor de quantidades desconhecidas, tem componentes $\theta_{1}, \theta_{2}, \ldots, \theta_{k}$. Seja $\pi\left(\theta_{i} \mid \boldsymbol{\theta}_{-i}, \boldsymbol{y}\right), \quad i=1, \ldots, k$, a distribuição condicional completa para todos os componentes individuais do vetor de quantidades desconhecidas $\boldsymbol{\theta}$, dadas as observações. Valores especificados para todos os outros componentes $\boldsymbol{\theta}$ são facilmente identificados, como função de $\theta_{i}$ pela inspeção da forma $\pi(\boldsymbol{\theta} \mid \boldsymbol{y}) \propto \pi(\boldsymbol{y} \mid \boldsymbol{\theta}) \pi(\boldsymbol{\theta})$ em qualquer aplicação dada.

Suponha que dado um conjunto de pontos iniciais, $\theta_{1}^{(0)}, \theta_{2}^{(0)}, \ldots, \theta_{k}^{(0)}$ para uma quantidade desconhecida, implementa-se o seguinte procedimento iterativo:

i) Gerar $\theta_{1}^{(1)}$ da densidade condicional cheia $\pi\left(\theta_{1} \mid \theta_{2}^{(0)}, \ldots, \theta_{k}^{(0)}, \boldsymbol{y}\right)$

ii) Gerar $\theta_{2}^{(1)}$ da densidade condicional cheia $\pi\left(\theta_{2} \mid \theta_{1}^{(1)}, \theta_{3}^{(0)}, \ldots, \theta_{k}^{(0)}, \boldsymbol{y}\right)$

k) Gerar $\theta_{k}^{(1)}$ de $\pi\left(\theta_{k} \mid \theta_{1}^{(1)}, \theta_{2}^{(1)}, \ldots, \theta_{k-1}^{(1)}, \boldsymbol{y}\right)$

e iteragir o processo $t$ vezes.

Executando os passos de $(i)-(k) t$ vezes, segundo Geman \& Geman (1984), obtém-se um vetor de amostras $\boldsymbol{\theta}^{t}=\left(\theta_{1}^{t}, \ldots, \theta_{k}^{t}\right)$, em que $\boldsymbol{\theta}$ é uma realização da cadeia de Markov, sendo o primeiro elemento amostral da distribuição 
conjunta $\pi\left(\theta_{1}, \theta_{2}, \ldots, \theta_{k} \mid \boldsymbol{y}\right)$, isto é, o ponto $k$-dimensional produzido na $t$-ésima iteração do esquema amostral converge em distribuição para a variável aleatória cuja distribuição a posteriori é dada por $\pi\left(\theta_{1}, \theta_{2}, \ldots, \theta_{k} \mid \boldsymbol{y}\right)$ para um $t$ suficientemente grande. Além disso, $\theta_{i}^{(t)}$ pode ser considerado como uma observação simulada da distribuição marginal a posteriori de $\theta_{i}, \pi\left(\theta_{i} \mid \boldsymbol{y}\right)$.

\subsection{5 "Slice sampling"}

Outra alternativa para amostrar de distribuições condicionais completas a posteriori que não possuem forma fechada é o método "Slice sampling" proposto por Neal (2003).

O método consiste em amostrar de uma distribuição para uma variável $\theta$, cuja função densidade de probabilidade é proporcional a $f(\theta)$. Isso pode ser feito introduzindo uma variável auxiliar $\psi$, e definindo a distribuição conjunta de $\theta$ e $\psi$ a qual é uniforme sobre a região $U=\{(\theta, \psi): 0<\psi<f(\theta)\}$, abaixo da curva definida por $f(\theta)$. Então, a função densidade conjunta para $(\theta, \psi)$ é dada por:

$$
p(\theta, \psi)= \begin{cases}\frac{1}{Z} & \text { se } 0<\psi<f(\theta) \\ 0 & \text { caso contrário }\end{cases}
$$

sendo $Z=\int f(\theta) d \theta$, e a densidade marginal de $\theta$ dada por:

$$
p(\theta)=\int_{0}^{f(\theta)} \frac{1}{Z} d y=\frac{f(\theta)}{Z} .
$$

Para amostrar de $\theta$ basta amostrar conjuntamente $(\theta, \psi)$ e ignorar $\psi$.

Gerar $(\theta, \psi)$ conjuntamente amostrando uniformemente de $\mathrm{U}$ pode ser muito difícil, e para resolver o problema, Neal (2003) propôs um método alternativo para amostrar $\theta$ de qualquer distribuição contínua, sendo necessário apenas calcular algumas funções $f(\theta)$ proporcionais a densidade de $\theta$, seguindo as etapas:

i) Iniciar $\theta_{0}$ pertencente ao suporte de $f\left(\theta_{0}\right)$. 
ii) Amostrar um valor $\psi$, uniformemente de $\left(0, f\left(\theta_{0}\right)\right)$, definindo uma fatia horizontal $S=\{\theta: \psi<f(\theta)\}$, observe que $\theta_{0} \in S$.

iii) Encontrar um intervalo $I=(L, R)$, ao redor de $\theta_{0}$ que contém a maior parte da fatia.

iv) Amostrar um novo ponto, $\theta_{1}$, da parte da fatia dentro deste intervalo, ou seja, $\theta_{1} \in I \cap S$.
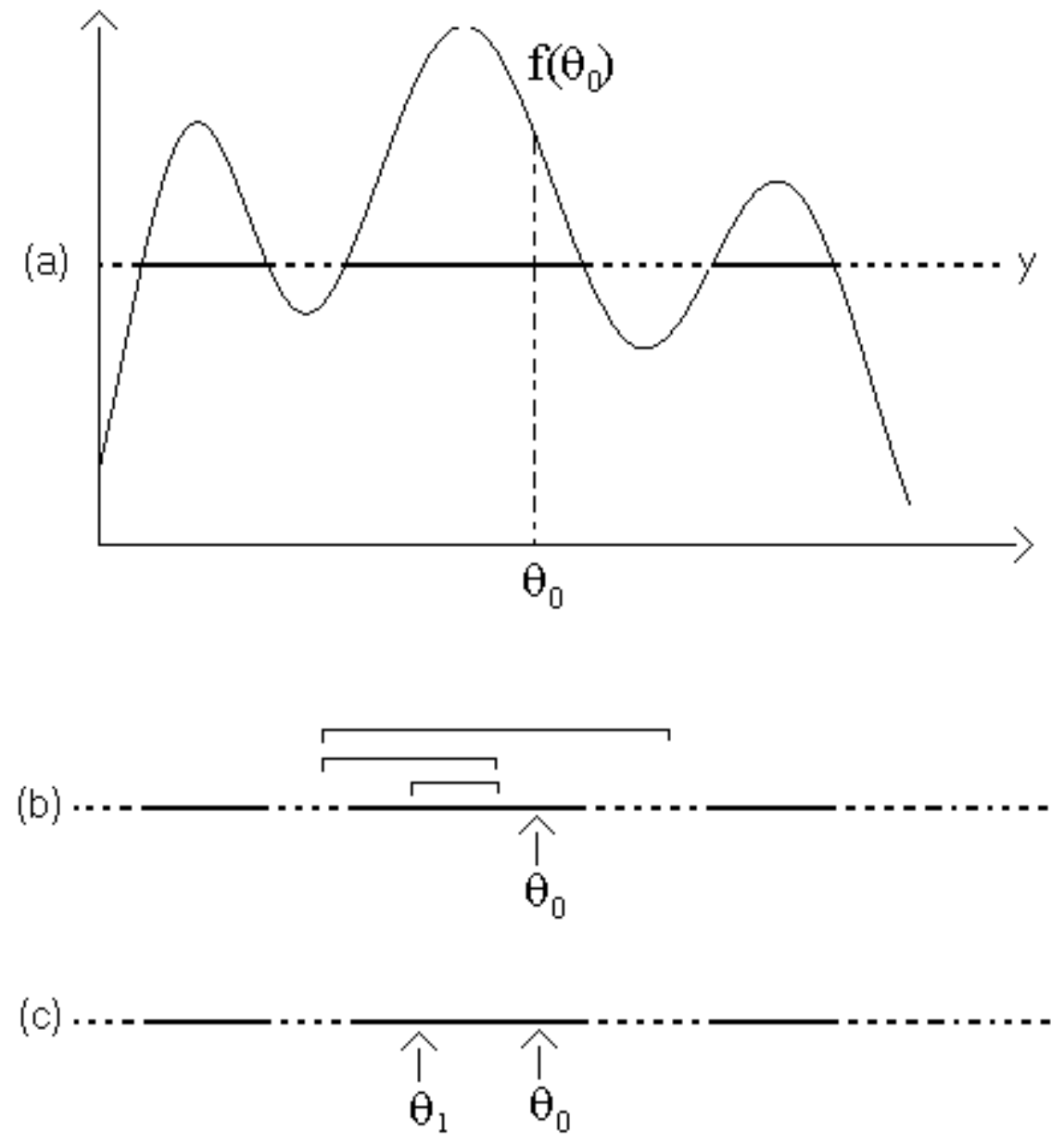

Figura 2 - "Slice sampling" 
A Figura 2 ilustra os passos acima. Um nível vertical, $\psi$, é amostrado uniformemente de $\left(0, f\left(\theta_{0}\right)\right)$ e usado para definir uma fatia horizontal indicada em negrito $(a)$. Um intervalo inicial de tamanho $r$ é aleatoriamente posicionado ao redor de $\theta_{0}$, e então dobra-se o tamanho de $r$ até que ambos os lados do intervalo estejam fora da fatia $(b)$. Um novo ponto, $\theta_{1}$, que pertence a fatia, é então encontrado $(c)$.

De forma equivalente, implementa-se o seguinte procedimento iterativo:

i) Inicie $\theta_{0}$;

ii) Amostre $\psi$ uniformente de $\left(0, f\left(\theta_{0}\right)\right)$;

$\psi \sim \operatorname{Uniforme}\left(0, f\left(\theta_{0}\right)\right)$

iii) Defina $r$, defina o limite do intervalo, $2^{\nu} r$, e encontre o intervalo $I=(L, R)$

$U \sim \operatorname{Uniforme}(0,1)$

$L \leftarrow \theta_{0}-r * U$

$R \leftarrow L+r$

$K \leftarrow \nu$

Repita:

Enquanto $K>0$ e $\{\psi<f(L)$ ou $\psi<f(R)\}$ :

$V V \sim$ Uniforme $(0,1)$

Se $V V<\frac{1}{2}$ então $L \leftarrow L-(R-L)$

senão $R \leftarrow R+(R-L)$

$K \leftarrow K-1$

iv) Encontre um novo ponto, $\theta_{1}$, pertencente a fatia e ao intervalo:

$\bar{L} \leftarrow L$

$\bar{R} \leftarrow R$

Repita:

$U \sim U$ ififorme $(0,1)$

$\theta_{1} \leftarrow \bar{L}+U(\bar{R}-\bar{L})$ 
Se $\psi<f\left(\theta_{1}\right)$ e $\operatorname{Aceitação~}\left(\theta_{1}\right)=\theta_{1}$ pare o processo

Se $\theta_{1}<\theta_{0}$ então $\bar{L} \leftarrow \theta_{1}$

senão $\bar{R} \leftarrow \theta_{1}$

v) Aceitação de $\theta_{1}$ :

$\hat{L} \leftarrow L$

$\hat{R} \leftarrow R$

$C \leftarrow$ falso

Repita:

Enquanto $\hat{R}-\hat{(} L)>1,1 r$ :

$M \leftarrow(\hat{L}+\hat{R}) / 2$

Se $\left\{\theta_{0}<M\right.$ e $\left.\theta_{1} \geq M\right\}$ ou $\left\{\theta_{0} \geq M\right.$ e $\left.\theta_{1}<M\right\}$ então $C \leftarrow$ verdadeiro

Se $\theta_{1}<M$ então $\hat{R} \leftarrow M$

senão $\hat{L} \leftarrow M$

Se $C=$ falso e $\psi \geq f(\hat{L})$ e $\psi \geq f(\hat{R})$ então $\theta_{1} \leftarrow \theta_{0}$

senão $\theta_{1} \leftarrow \theta_{1}$

E então, o novo ponto $\theta_{1}$ é amostrado.

\subsection{Conceito de Firma}

Firmas são instituições que coordenam a transformação de insumos em produtos (Nicholson, 2002), também organizam o local de trabalho e transformam recursos escassos em produtos e serviços que são vendidos nos mercados (Thompson \& Formby, 1998).

Em uma economia de mercado, a firma é o principal agente econômico para a produção e distribuição de bens e serviços. As firmas economizam em custos de transação e, mais do que qualquer outra unidade econômica, servem como o centro de atividade econômica. Elas investem em máquinas mais eficientes, desenvolvem 
novas tecnologias, vendem novos e melhores produtos, antecipam quais os tipos de insumos que serão utilizados nas atividades de produção e servem como a fonte principal de empregos e renda.

As firmas podem ser grandes instituições, como a General Motors, ou pequenas, como fazendas, lacticínios. Existem pelo menos seis tipos diferentes de firmas:

i) a empresa que opera em um único ramo de atividade e em um único estágio da cadeia de produção de um setor;

ii) a empresa parcialmente verticalizada que opera em um único ramo de atividade e em alguns estágios da cadeia de produção de um setor;

iii) a empresa totalmente verticalizada que opera em único ramo de atividade e em todos os estágios da cadeia de produção;

iv) a "empresa dominante" que possui vendas concentradas em um dos principais setores da economia, mas com um portfólio de négocios em setores relacionados ou não, modestamente diversificado (respondendo por um terço ou menos) das vendas totais da corporação;

v) a empresa limitadamente diversificada que possui algumas (de 3 a 10) unidades operando em setores relacionados ou não-relacionados, e

vi) a empresa amplamente diversificada com muitas (entre 10 e várias centenas) unidades operando em uma variedade de setores relacionados ou nãorelacionados.

Na prática, há grande diversidade entre as firmas: elas podem ser de propriedade pública ou privada, podem ter fins lucrativos, ou não, e podem ser organizadas como corporações, sociedades anônimas ou limitadas.

O papel econômico das firmas é tão importante que a eficiência com que desempenham suas atividades torna-se um determinante central do bem-estar 
econômico da sociedade, e para determinar essa eficiência é necessário considerar toda essa diversidade.

\subsection{Função de produção}

Uma função de produção (Figura 3) relaciona insumos $x$ e produtos $y$ de um processo de produção, definindo o produto máximo conseguido através de um dado vetor de insumos (Coelli et al., 1999). Insumos podem ser materiais usados na produção de algum bem, e produtos o que é obtido usando quantidades de insumos.

Essa relação pode ser expressa da seguinte forma:

$$
y=f(\boldsymbol{x})
$$

sendo $y \in \Re$ o produto, e $\boldsymbol{x} \in \Re^{S}$ vetor de $S$ insumos.

As relações de insumo-produto dependem em parte das quantidades de recursos empregados e, em parte, da forma pela qual esses insumos são combinados (a tecnologia de produção empregada pela firma).

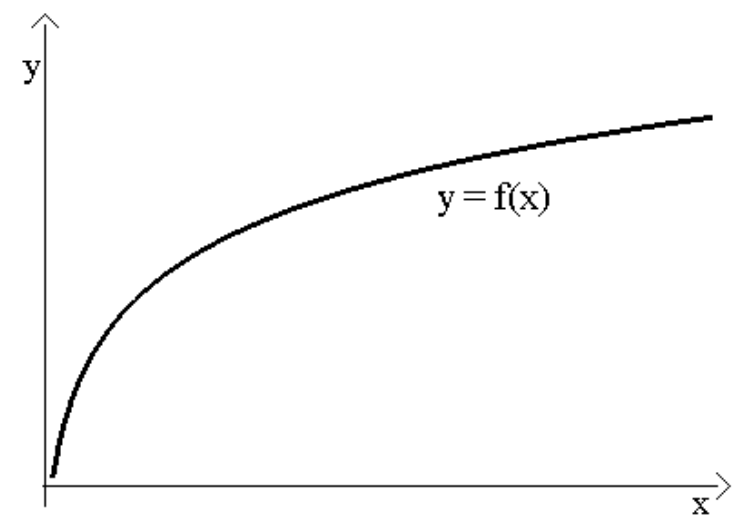

Figura 3 - Função de produção

As funções de produção são, geralmente, representadas por funções matemáticas, chamadas formas funcionais e as mais conhecidas são Cobb-Douglas e 
Translog.

\subsubsection{Cobb-Douglas}

A forma funcional Cobb-Douglas (Figura 4) é a mais utilizada (Coelli et al., 1999). Considerando o caso com um único produto $y$ e dois insumos $x_{1}, x_{2}$, a forma funcional Cobb-Douglas pode ser expressa como:

$$
y=A x_{1}^{\beta_{1}} x_{2}^{\beta_{2}}
$$

sendo $\beta_{1}$ e $\beta_{2}$ parâmetros positivos, os quais medem a elasticidade do produto, isto é, a elasticidade parcial $\beta_{1}$ do produto com respeito ao insumo $x_{1}$, mede a variação percentual em $y$ resultante do aumento de $1 \%$ em $x_{1}$, mantendo $x_{2}$ constante, e a elasticidade parcial $\beta_{2}$ do produto com respeito ao insumo $x_{2}$, mede a variação percentual em $y$ resultante do aumento de $1 \%$ em $x_{2}$, mantendo $x_{1}$ constante (Gujarati, 1995).

De forma equivalente, tem-se:

$$
\ln (y)=\beta_{0}+\beta_{1} \ln \left(x_{1}\right)+\beta_{2} \ln \left(x_{2}\right) .
$$

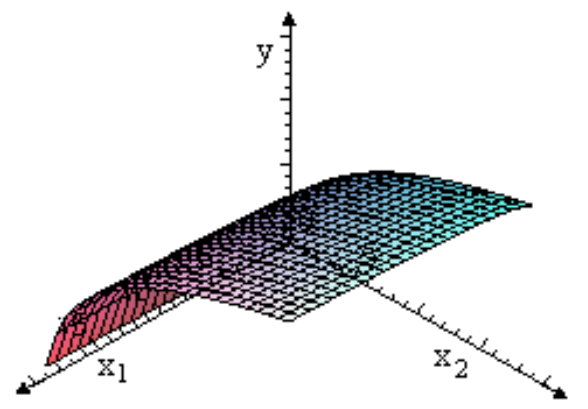

Figura 4 - Forma funcional Cobb-Douglas 
A estimação dos parâmetros da função de produção (6) não é uma tarefa muito difícil e nem sua manipulação matemática, no entanto, as propriedades impostas pela estrutura de produção exige retorno constante à escala, $\beta_{1}+\beta_{2}=1$.

\subsubsection{Translog}

A forma funcional Translog (Figura 5) é dada por:

$$
y=\exp \left\{\beta_{0}+\sum_{n=1}^{S} \beta_{n} \ln \left(x_{n}\right)+\frac{1}{2} \sum_{m=1}^{S} \sum_{n=1}^{S} \beta_{m n} \ln \left(x_{m}\right) \ln \left(x_{n}\right)\right\} .
$$

De forma equivalente, considerando o caso de um único produto y e dois insumos $x_{1}, x_{2}$, tem-se:

$$
\ln (y)=\beta_{0}+\beta_{1} \ln \left(x_{1}\right)+\beta_{2} \ln \left(x_{2}\right)+\frac{1}{2}\left[\beta_{11} \ln ^{2}\left(x_{1}\right)+\beta_{22} \ln ^{2}\left(x_{2}\right)\right]+\beta_{12} \ln \left(x_{1}\right) \ln \left(x_{2}\right) .
$$

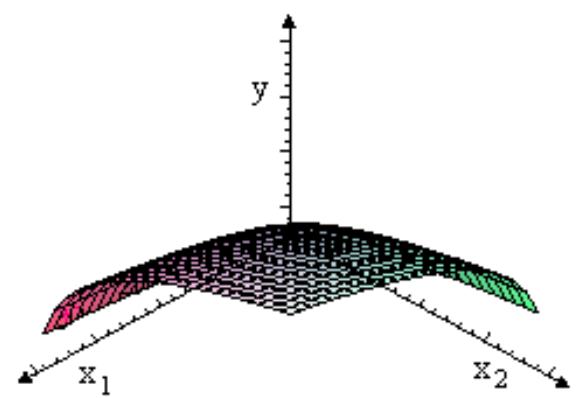

Figura 5 - Forma funcional Translog

A forma funcional Translog não impõe restrições sobre a estrutura de produção. Não há necessidade de considerar retorno à escala constante, mas é mais difícil de manipular algebricamente e pode sofrer com problemas de multicolinearidade e graus de liberdade (Coelli et al., 1999).

A forma funcional Cobb-Douglas (6) é um caso especial da forma funcional Translog (8). 


\subsubsection{Retornos à escala}

Retornos à escala refletem o grau em que um aumento proporcional em todas as quantidades de insumos, aumenta as quantidades de produtos (Coelli et al., 1999). Podem ocorrer retornos à escala constantes, crescentes e decrescentes:

i) retornos à escala constantes ocorrem quando um aumento proporcional em todos os insumos resulta no mesmo aumento nos produtos, assim, dobrando-se a quantidade de insumos, obtém-se o dobro das quantidades de produtos;

ii) retornos à escala crescentes ocorrem quando um aumento proporcional em todos os insumos resulta em um aumento maior nos produtos, assim, dobrando-se os insumos, os produtos são maiores do que o dobro, e

iii) retornos à escala decrescentes ocorrem quando um aumento proporcional em todos os insumos resulta em um aumento menor nos produtos, assim, dobrando-se os insumos, os produtos são menores do que o dobro.

De outra forma, considerando a tecnologia de produção $y=f\left(x_{1}, x_{2}\right)$, tem-se:

$$
t^{r} y=f\left(t x_{1}, t x_{2}\right),
$$

sendo $t \in \Re$ uma constante e $r$ o grau de homogeneidade da função. Quando $r>1$, caracteriza-se retorno à escala crescente, quando $r<1$, caracteriza-se retorno à escala decrescente e quando $r=1$ caracteriza-se retorno à escala constante.

\subsubsection{Produto Marginal}

Quando se deseja verificar a variação dos produtos em que apenas um dos insumos é alterado usa-se o produto marginal. O produto marginal está associado às derivadas parciais da função de produção (Medrano, 2003). 
Considerando uma função de produção com um único produto y e um insumo qualquer $x_{j}$, o produto marginal em relação ao insumo $x_{j}$ é definido como:

$$
P M_{j}=\frac{\partial f(x)}{\partial x_{j}}
$$

O produto marginal positivo acontece quando aumentando a quantidade de um insumo mantendo os outros constantes obtêm-se rendimentos crescentes na produção, quando ocorrer rendimentos decrescentes o produto marginal será negativo.

\subsection{Fronteira de produção}

Fronteiras de produção são funções de produção (Cobb-Douglas, Translog etc) com componente aleatório (Pinilla, 2001). Podem ser determinísticas ou estocásticas.

\subsubsection{Fronteira de produção determinística}

Fronteira de produção determinística (Figura 6) pode ser escrita como:

$$
y=f(x)-u
$$

sendo $u$ uma variável aleatória com distribuição assimétrica, assumindo valores maiores ou iguais a zero.

As fronteiras de produção determinísticas possuem apenas uma variável aleatória, devido ao termo de ineficiência da firma, ignorando a natureza estocástica da produção, ou seja, toda a distância da firma à fronteira deve-se a sua ineficiência. 


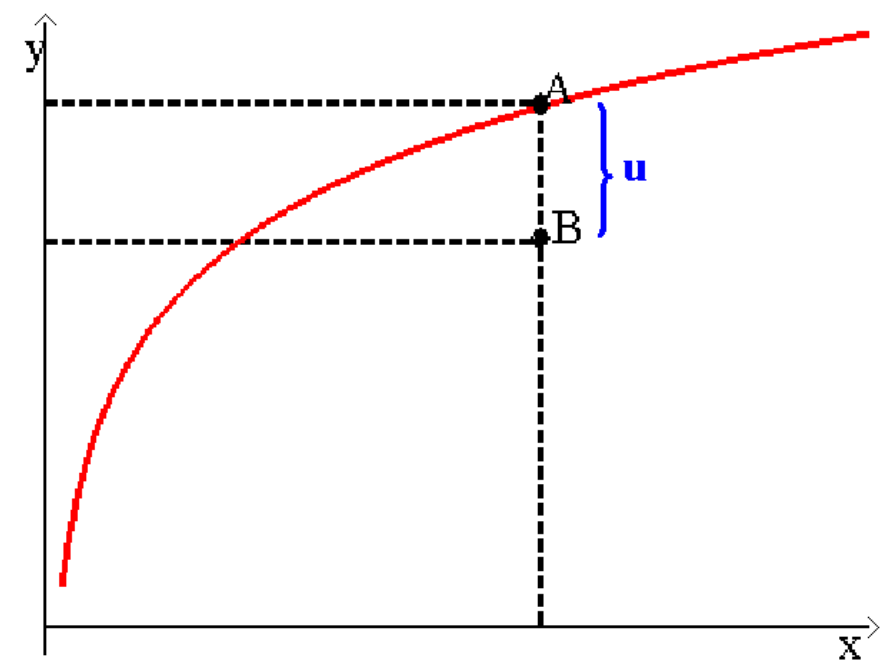

Figura 6 - Fronteira de Produção Determinística

\subsubsection{Fronteira de produção estocástica}

Fronteira de produção estocástica (Figura 7) pode ser escrita da seguinte forma:

$$
y=f(x)+v-u
$$

sendo $v$ uma variável aleatória com distribuição simétrica, que se supõe idêntica e independentemente distribuída, com média zero e variância constante, e $u$ uma variável aleatória com distribuição assimétrica assumindo valores maiores ou iguais a zero, independente de $v$.

O componente $v$ representa o que não pode ser controlado pelas firmas, a flutuação natural, e $u$ a medida de ineficiência técnica na produção da quantidade do produto $y$ considerando as quantidades $x_{1}, x_{2}, \ldots, x_{S}$ de insumos. 


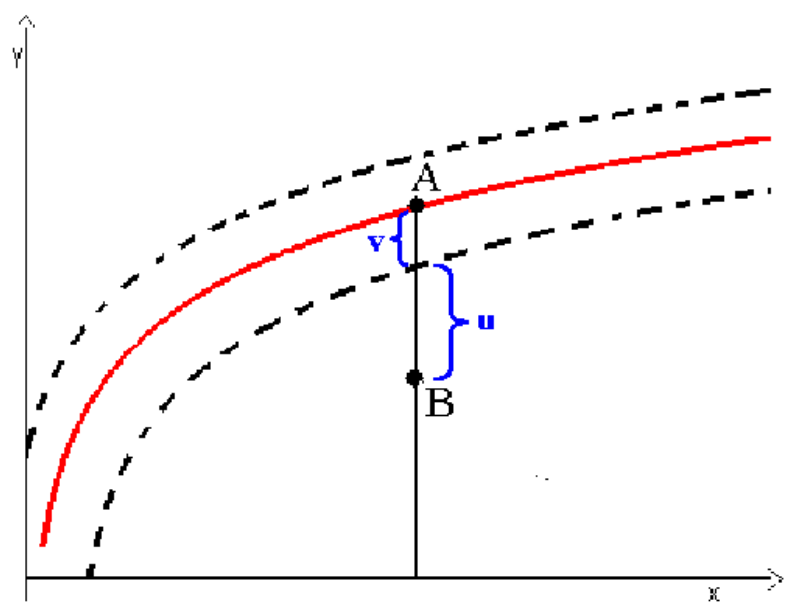

Figura 7 - Fronteira de Produção Estocástica

\subsection{Eficiência Produtiva}

As empresas que maximizam seus lucros são consideradas eficientes (Pinilla, 2001). Para uma empresa ser eficiente é preciso tomar corretamente três decisões:

i) dentre todos os níveis de produção possíveis, escolher o produto que maximize o lucro;

ii) dentre todas as combinações de insumos que servem para produzir o nível de produto máximo, a empresa deve escolher aquela combinação de insumos que minimize o custo de produção, e

iii) a empresa deve produzir o produto escolhido com a quantidade mínima de insumos possíveis, ou seja, deve utilizar sua tecnologia corretamente.

Portanto, pode-se falar em três tipos de eficiência:

i) eficiência de escala: quando a empresa produz uma quantidade para a qual o ingresso marginal é igual ao custo marginal; 
ii) eficiência alocativa: quando o valor do produto marginal de cada fator for igual ao seu preço, e

iii) eficiência técnica: quando a empresa trabalha na fronteira de produção.

\subsubsection{Eficiência técnica}

Firmas que operam na fronteira de produção são tecnicamente eficientes e se operam abaixo da fronteira são tecnicamente ineficientes. Considerando uma tecnologia de produção simples, com um insumo $x$ e um produto $y$ (Figura 8), tem-se que a firma $\mathrm{A}$ é mais eficiente do que a firma B, pois com a mesma quantidade de insumos, a firma A produz mais do que a firma B.

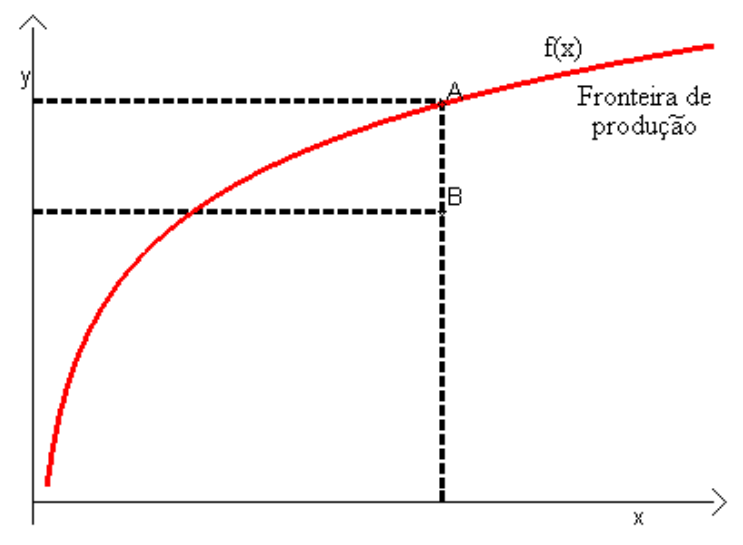

Figura 8 - Eficiência

Não se pode confundir eficiência técnica com produtividade, pois os conceitos não têm nenhuma relação quando variam os insumos e produtos. A Figura 9 ilustra a diferença entre eficiência técnica e produtividade. Produtividade é a razão de produtos que são obtidos com os insumos utilizados. A reta partindo da origem e passando por um ponto particular, representando uma determinada firma, mede a produtividade dessa firma. A inclinação desta reta $\frac{y}{x}$ produz a medida de produtividade. 


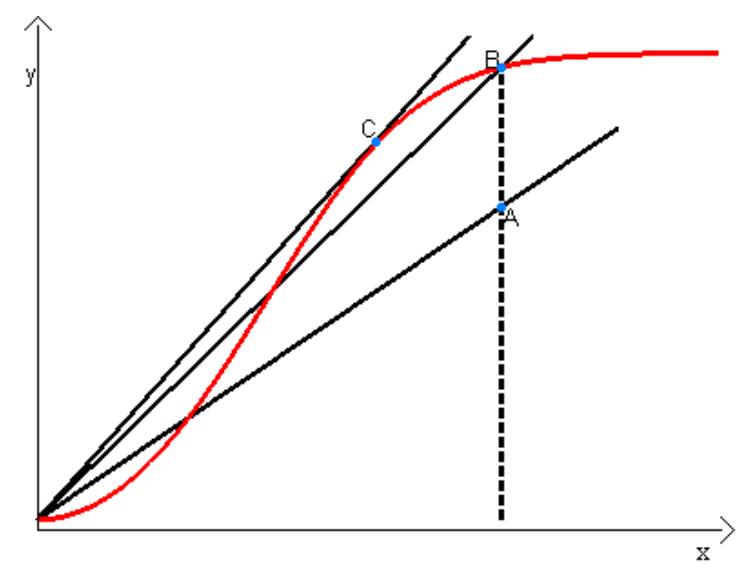

Figura 9 - Produtividade e Eficiência técnica

Se a firma $A$ mover-se para a eficiência técnica da firma $B$, a inclinação da reta será maior, implicando maior produtividade em $B$. Contudo, a reta passando pela firma $C$ é a tangente à fronteira de produção, definindo o ponto de produtividade máxima possível, qualquer outro ponto resulta em menor produtividade (Coelli et al., 1999), o que mostra que uma firma pode se tornar eficiente mas menos produtiva.

A eficiência de uma firma pode ser estudada de várias maneiras e dependendo do tipo de eficiência a ser calculada são necessários conjunto de dados diferentes. Se o objetivo for comparar a eficiência de uma mesma firma ao longo do tempo, dados de insumos e produtos devem ser coletados em $T$ diferentes tempos, como mostra, esquematicamente, a Tabela 1:

Tabela 1. Séries temporais

\begin{tabular}{cccccc}
\hline \multicolumn{7}{c}{ Firma 1 } \\
\hline$t_{1}$ & $x_{11}$ & $x_{12}$ & $\ldots$ & $x_{1 S}$ & $y_{1}$ \\
$t_{2}$ & $x_{21}$ & $x_{22}$ & $\ldots$ & $x_{2 S}$ & $y_{2}$ \\
$\ldots$ & $\ldots$ & $\ldots$ & $\ldots$ & $\ldots$ & $\ldots$ \\
$t_{T}$ & $x_{T 1}$ & $x_{T 2}$ & $\ldots$ & $x_{T S}$ & $y_{T}$ \\
\hline
\end{tabular}


sendo $x_{i j}$ e $y_{i}$, os insumos e os produtos de uma firma com $i=1, \ldots, T$ tempos e $j=1, \ldots, S$ insumos.

Se o objetivo for comparar a eficiência de uma determinada firma com outras, os dados das firmas devem ser coletados no mesmo período de tempo, ou seja, dados de insumos e produtos de todas as $N$ firmas devem ser coletados no mesmo período $T$ (Tabela 2):

Tabela 2. "Cross-sectional"

\begin{tabular}{ccccc}
\hline & Firma 1 & Firma 2 & $\ldots$ & Firma N \\
\hline & $x_{11}$ & $x_{12}$ & $\ldots$ & $x_{1 N}$ \\
& $x_{21}$ & $x_{22}$ & $\ldots$ & $x_{2 N}$ \\
$T$ & $\vdots$ & $\vdots$ & $\vdots$ & $\vdots$ \\
& $x_{S 1}$ & $x_{S 2}$ & $\ldots$ & $x_{S N}$ \\
& $y_{1}$ & $y_{2}$ & $\ldots$ & $y_{N}$ \\
\hline
\end{tabular}

sendo $x_{j k}$ e $y_{k}$, os insumos e os produtos das firmas com $j=1, \ldots, S$ insumos e $k=1, \ldots, N$ firmas.

Se o objetivo for comparar a eficiência de várias firmas em vários tempos, os dados das firmas devem ser coletados em vários tempos, ou seja, dados de insumos e produtos de todas as $N$ firmas devem ser coletados nos $T$ tempos (Tabela 3):

Tabela 3. Painel

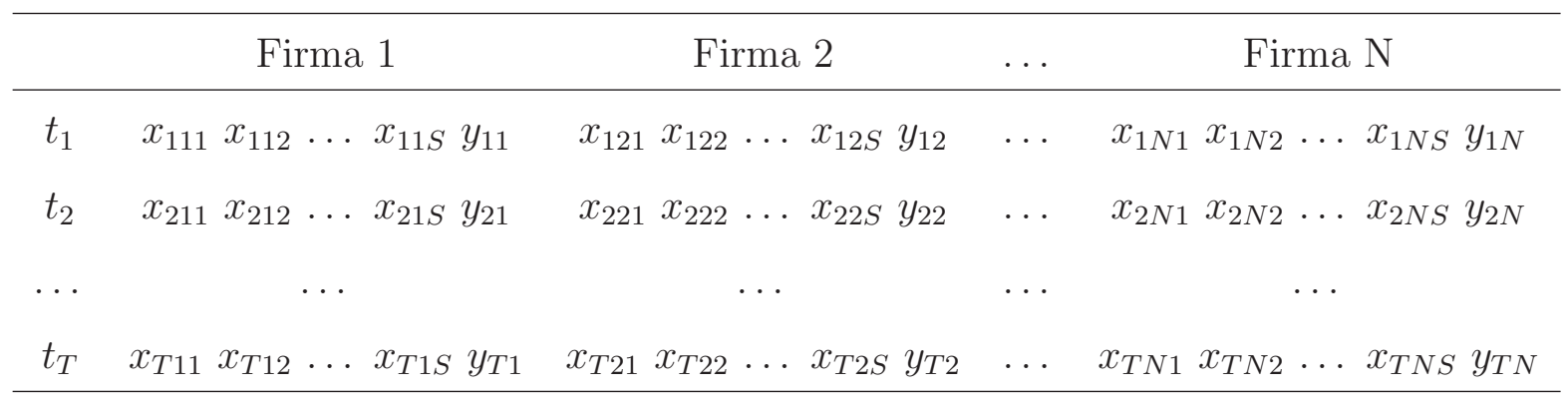


sendo $x_{i k j}$ e $y_{i k}$, os insumos e os produtos das firmas com $i=1, \ldots, T$ tempos, $k=1, \ldots, N$ firmas e $j=1, \ldots, S$ insumos.

\subsubsection{Modelando os efeitos de ineficiência}

Os primeiros a modelar a fronteria de produção determinística foram Aigner \& Chu (1968), considerando a estimativa de uma fronteira paramétrica da função de produção Cobb-Douglas, os quais, propuseram o modelo:

$$
\ln \left(y_{i}\right)=x_{i} \beta-u_{i}, \quad i=1, \ldots N
$$

sendo $\ln \left(y_{i}\right)$ o logaritmo do produto para a i-ésima firma; $x_{i}$ um vetor de dimensão $(k+1)$, com o primeiro elemento igual a 1 e os restantes logaritmos dos $k$ insumos usados pela i-ésima firma; $\boldsymbol{\beta}=\left(\beta_{0}, \beta_{1}, \ldots, \beta_{k}\right)^{\prime}$ um vetor de parâmetros desconhecidos a serem estimados; e $u_{i}$ é uma variável aleatória com distribuição não-negativa, associada à ineficiência técnica na produção das firmas.

De forma equivalente tem-se:

$$
y_{i}=f\left(x_{i}\right) \exp \left(-u_{i}\right), \quad i=1, \ldots N
$$

A razão dos produtos observados pela i-ésima firma, relativa ao produto definido pela fronteira de produção, dado o vetor de insumos, $x_{i}$, é usada para definir a eficiência técnica da i-ésima firma:

$$
E T_{i}=\frac{\exp \left(x_{i} \boldsymbol{\beta}-u_{i}\right)}{\exp \left(x_{i} \boldsymbol{\beta}\right)}=\exp \left(-u_{i}\right),
$$

que assume valores entre zero e um. A equação (10) indica a magnitude do produto da i-ésima firma relativa ao produto que poderia ser obtido por uma firma eficiente usando o mesmo vetor de insumos. Uma crítica a esse modelo é que todos os desvios da fronteira são assumidos como resultado da ineficiência técnica, ou seja, garante que a medida de eficiência assume que a função de produção da firma é conhecida, mas que na realidade nunca o é (Coelli et al., 1999). 
Assim, Aigner et al. (1977) e Meeusen \& van den Broeck (1977), independentemente, propuseram a fronteira de produção estocástica (Figura 10) na qual um erro aleatório adicional, $v_{i}$, é somado a uma variável aleatória não-negativa, $u_{i}$, na equação (9) obtendo:

$$
y_{i}=f\left(x_{i}\right) \exp \left(v_{i}-u_{i}\right), \quad i=1, \ldots N
$$

O erro aleatório, $v_{i}$, considera a medida de erro e outros fatores aleatórios, tais como, efeitos de tempo, greves, sorte etc, sobre os valores dos produtos, junto com os efeitos combinados de insumos não-especificados na função de produção. Aigner et al. (1977) assumem que os $v_{i}$ são variáveis aleatórias independentes e identicamente distribuídas com distribuição normal, com média zero e variância constante, independentes $\operatorname{dos} u_{i}^{\prime} s$, que são variáveis aleatórias independentes e identicamente distribuídas com distribuição exponencial ou meia normal. Poucos pesquisadores, como Stevenson (1980) e Greene (1990) especificaram uma forma mais geral para a distribuição dos efeitos da ineficiência técnica. Stevenson (1980) especificou a distribuição normal truncada e Greene (1990) usou a distribuição gama com dois parâmetros.

E então, a eficiência técnica é medida como (Cuesta et al., 2001):

$$
E T_{i}=\frac{f\left(x_{i}\right) \exp \left(v_{i}-u_{i}\right)}{f\left(x_{i}\right) \exp \left(v_{i}\right)}=\exp \left(-u_{i}\right),
$$

sendo $f\left(x_{i}\right)$ uma função de insumos, $v_{i}$ uma variável aleatória representando o erro aletatório e $u_{i}$ variável aleatória representando a ineficiência da firma.

Um dos primeiros artigos sobre modelos de fronteira estocástica utilizando a abordagem Bayesiana foi apresentado por van den Broeck et al. (1994) no qual a distribuição gama com parâmetro de forma conhecida é adotada para o termo de ineficiência.

Outro trabalho utilizando a abordagem Bayesiana proposto por Koop et al. (1995) utiliza o amostrador de Gibbs para analisar o modelo de fronteira estocástica com distribuição gama para a ineficiência. Fernández et al. (1997) e 


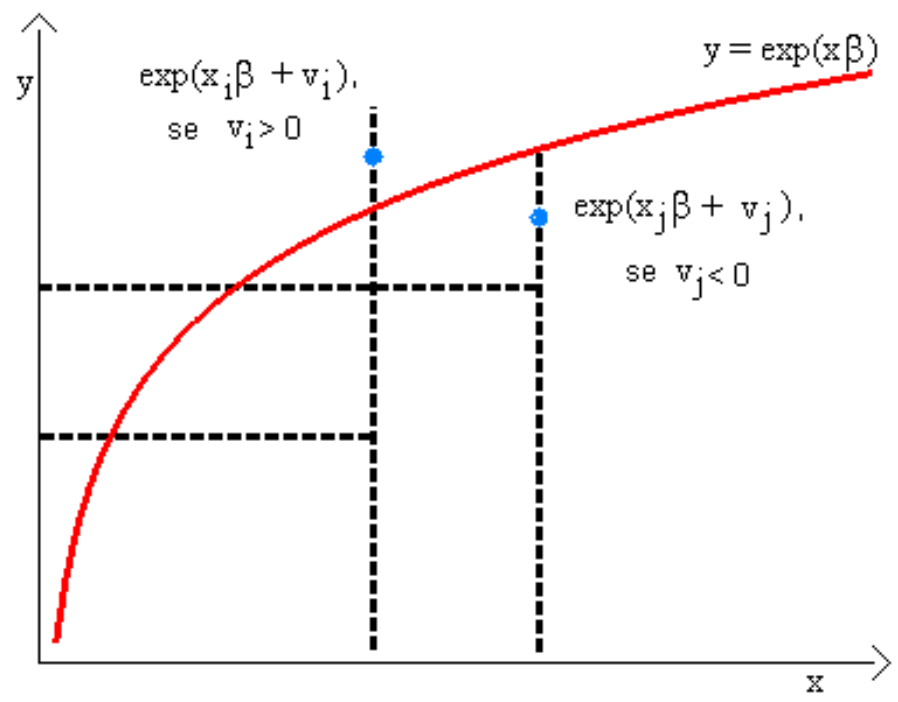

Figura 10 - Fronteira de Produção

Koop et al. (1997) propõem modelos para dados em painel no contexto Bayesiano, Osiewalski \& Steel (1998) discutem aspectos numéricos na análise Bayesiana para modelos de fronteira estocástica. Koop \& Steel (2001) apresentam uma revisão recente da abordagem Bayesiana para fronteiras estocásticas.

Tsionas (2000), defrontando-se com alguns parâmetros que não possuem distribuição condicional a posteriori completa conhecida, propõe um método de aceitação para gerar valores de distribuições condicionais completas. Tsionas (2002) propõe um modelo de fronteira estocástica com coeficientes aleatórios para separar a ineficiência técnica de diferenças tecnológicas de firmas, libertando o modelo de fronteira da suposição restritiva que todas as firmas tenham que compartilhar as mesmas possibilidades tecnológicas.

Também em 2002, Migon² propõe utilizar a distribuição log-normal para o termo de ineficiência da firma.

$\mathrm{Na}$ abordagem clássica alguns estudos têm pesquisado determinantes

\footnotetext{
${ }^{2}$ MIGON, H.S. A Bayesian approach to stochastic production frontier. migon@im.ufrj.br
} (10 Março 2003) 
de ineficiência técnica entre firmas pela regressão de efeitos de ineficiência preditos, obtidos de uma fronteira estocástica estimada, sobre um vetor de fatores específicos de cada firma, tais como tamanho, idade etc (Coelli et.al., 1999). Há um problema significante com essa abordagem: num primeiro estágio, os efeitos de ineficiência são independentes e identicamente distribuídos para predizer os valores dos efeitos de ineficiência técnica, contudo, num segundo estágio, os efeitos de ineficiência preditos assumem ser uma função de um número de fatores específicos de cada firma, o que implica que não são mais identicamente distribuídos. Fernández et al. (2000) resolve o problema utilizando a abordagem Bayesiana, incorporando no modelo, através de uma estrutura hierárquica, essas variáveis "exógenas" (tamanho, idade etc). 


\section{MATERIAL E MÉTODOS}

\subsection{Material}

O material usado nesse trabalho será um conjunto de dados simulados. Os dados são gerados para 5 firmas em 12 tempos, considerando-se que cada firma possui 3 produtos e um insumo, e possuem a seguinte estrutura:

Firma 1 $\quad\left[\begin{array}{cccc}t_{1} & t_{2} & \ldots & t_{12} \\ y_{1,1,1} & y_{1,2,1} & & y_{1,12,1} \\ y_{1,1,2} & y_{1,2,2} & \ldots & y_{1,12,2} \\ y_{1,1,3} & y_{1,2,3} & & y_{1,12,3} \\ y_{2,1,1} & y_{2,2,1} & & y_{2,12,1} \\ y_{2,1,2} & y_{2,2,2} & \ldots & y_{2,12,2} \\ y_{2,1,3} & y_{2,2,3} & & y_{2,12,3} \\ \vdots & \vdots & \vdots & \vdots \\ y_{5,1,1} & y_{5,2,1} & & y_{5,12,1} \\ y_{5,1,2} & y_{5,2,2} & \ldots & y_{5,12,2} \\ y_{5,1,3} & y_{5,2,3} & & y_{5,12,3}\end{array}\right]$




\subsection{Métodos}

\subsubsection{Modelo}

Quando se pretende analisar a eficiência técnica de firmas que possuem múltiplos produtos, através da fronteira de produção, é necessário construir um índice desses produtos com pesos fixos ou com pesos estimados (Fernández et al., 2000).

Considerando um conjunto com $N T$ observações correspondendo a $P$ produtos de $N$ firmas diferentes, sobre $T$ tempos, define-se o vetor de produtos de dimensão $P$, da firma $i$ no tempo $t$, por:

$$
y_{i, t}=\left(y_{i, t, 1}, y_{i, t, 2}, \ldots, y_{i, t, P}\right)
$$

sendo $i=1, \ldots, N$ e $t=1, \ldots, T$.

Considerando toda a produção, tem-se:

$$
\left[\begin{array}{cccc}
y_{1,1,1} & y_{1,2,1} & \cdots & y_{1, T, 1} \\
y_{1,1,2} & y_{1,2,2} & \cdots & y_{1, T, 2} \\
\vdots & \vdots & \ddots & \vdots \\
y_{1,1, P} & y_{1,2, P} & \cdots & y_{1, T, P} \\
& & & \\
\vdots & \vdots & \ddots & \vdots \\
& & & \\
y_{N, 1,1} & y_{N, 2,1} & \cdots & y_{N, T, 1} \\
y_{N, 1,2} & y_{N, 2,2} & \cdots & y_{N, T, 2} \\
\vdots & \vdots & \ddots & \vdots \\
y_{N, 1, P} & y_{N, 2, P} & \cdots & y_{N, T, P}
\end{array}\right]
$$


Quando uma firma possui múltiplos produtos, frequentemente esses produtos têm unidade de medida diferentes (ex. litros, hectares, kilogramas), o que impossibilita a análise conjunta desses produtos. Para a análise dos dados ser possível, é necessário criar um índice, através de uma transformação ponderada, que garanta uma escala comum para esses produtos. A transformação considerada neste trabalho será dada por:

$$
\theta_{i, t}=\left(\sum_{j=1}^{P} \alpha_{j}^{q} y_{i, t, j}^{q}\right)^{\frac{1}{q}}
$$

sendo $q>1$ a elasticidade da transformação dos produtos (Powell \& Gruen, 1968 e Kumbhakar, 1987) e $\alpha_{j} \in(0,1)$, para todo $j=1, \ldots, P$, a garantia da invariância da reescala do produto individual pois não se pode assegurar uma medida comum para todo componente $P$, sabendo que $\sum_{j=1}^{P} \alpha_{j}=1$.

Considerando toda a produção, tem-se:

$$
\left[\begin{array}{cccc}
\theta_{11} & \theta_{12} & \cdots & \theta_{1 T} \\
\theta_{21} & \theta_{22} & \cdots & \theta_{2 T} \\
\vdots & \vdots & \ddots & \vdots \\
\theta_{N 1} & \theta_{N 2} & \cdots & \theta_{N T}
\end{array}\right]
$$

Dessa maneira, a equação (11) define a transformação de um vetor de produtos multivariado $y_{i, t}$, em uma quantidade univariada $\theta_{i, t}$, que pode ser interpretada como um "produto agregado", ou seja, são todos os vetores de produtos $y_{i, t}$, de dimensão $P$, que são equivalentes tecnologicamente, e correspondem aos $\theta_{i, t}$ que definem uma superfície de dimensão $(P-1)$ pertencente a $\Re_{+}^{P}$.

Por problemas de escala faz-se uma transformação logarítmica nos produtos agregados, definindo-se o vetor $\delta_{i, t}=\log \left(\theta_{i, t}\right)$ de dimensão $N T$, ou seja,

$$
\boldsymbol{\delta}=\left(\delta_{1,1}, \delta_{1,2}, \ldots, \delta_{1, T}, \ldots, \delta_{N, T}\right)^{\prime}
$$


Modelando-se $\boldsymbol{\delta}$ a partir do modelo de fronteira estocástica proposto por Fernández et al. (2000), tem-se:

$$
\boldsymbol{\delta}=\boldsymbol{V} \boldsymbol{\beta}-\boldsymbol{D} \boldsymbol{z}+\sigma \varepsilon
$$

sendo $\boldsymbol{V} \boldsymbol{\beta}$ a forma funcional da fronteira de produção, $\boldsymbol{D} \boldsymbol{z}$ o termo de ineficiência das firmas e $\sigma \varepsilon$ o termo de erro aleatório do modelo, ou seja, pode-se dividir o modelo proposto na equação (12) em três partes:

i) Definição da forma funcional:

A matriz $\boldsymbol{V}=\left(v\left(x_{1,1}\right), \ldots, v\left(x_{N, T}\right)\right)^{\prime}$, de dimensão $N T \times k$, a qual, especifica a forma funcional da fronteira de produção a ser estimada, através de $v\left(x_{i, t}\right)$, que é uma função dos insumos $x_{i, t}$ correspondente a firma $i$ no tempo t. Quando se considera a forma funcional Cobb-Douglas, $v\left(x_{i, t}\right)$ são os logaritmos dos insumos da firma $i$ no tempo $t$. Se a forma funcional translog é utilizada, consideram-se também os quadrados dos logaritmos dos insumos e seus produtos cruzados.

O vetor de dimensão $k$ dos coeficientes de regressão é denotado por $\boldsymbol{\beta}$, restrito a condições de regularidade que restringem o espaço paramétrico, sendo o intercepto estritamente maior que zero e os outros regressores maiores ou iguais a zero, para assegurar que os produtos marginais dos insumos sejam positivos, ou seja, que a produção tem rendimentos crescentes.

ii) Termo de ineficiência:

A ineficiência da firma é definida pelo vetor $\boldsymbol{\gamma}=\boldsymbol{D} \boldsymbol{z}$, sendo $\boldsymbol{D}$ uma matriz $N T \times M,(M \leq N T)$. Através da escolha de $\boldsymbol{D}$, definem-se diferentes vetores de ineficiência, constantes ou não no tempo. Se $\boldsymbol{D}$ é igual a matriz identidade de dimensão $N T$, obtém-se um termo de ineficiência diferente para cada firma $i$, em cada tempo $t$ : 


$$
\boldsymbol{D}=\left[\begin{array}{ccccc}
1 & 0 & 0 & \cdots & 0 \\
0 & 1 & 0 & \cdots & 0 \\
0 & 0 & 1 & \cdots & 0 \\
\vdots & \vdots & \vdots & \ddots & \vdots \\
0 & 0 & 0 & \cdots & 1
\end{array}\right]
$$

Se $\boldsymbol{D}$ é o produto de Kronecker entre a matriz identidade de dimensão $N$ e um vetor de uns $\left(\iota_{T}\right)$ de dimensão $T$, especifica-se um termo de ineficiência para cada firma $i, i=1, \ldots, N$ mas constante no tempo:

$$
\begin{aligned}
& {\left[\begin{array}{cccc}
1 & 0 & \cdots & 0 \\
1 & 0 & \cdots & 0 \\
\vdots & \vdots & \ddots & \vdots \\
1 & 0 & \cdots & 0
\end{array}\right.} \\
& \begin{array}{llll}
1 & 0 & \cdots & 0
\end{array} \\
& \begin{array}{llll}
1 & 0 & \cdots & 0
\end{array} \\
& \vdots \quad \vdots \quad \vdots \\
& \begin{array}{llll}
1 & 0 & \cdots & 0
\end{array}
\end{aligned}
$$

Existem outras possibilidades para a matriz D (Fernández et al., 1997).

iii) Erro aleatório:

O modelo considera que a fronteira não é conhecida realmente, portanto, possui um fator de escala $\sigma \in \Re_{+}$e uma perturbação aleatória $\varepsilon$, com distribuição 
normal $N T$ variada com média zero e matriz de variância e covariância a identidade de dimensão $N T$.

Considerando o caso mais simples, em que o termo de ineficiência seja específico para cada firma, mas constante no tempo, ou seja, tomando a matriz $\boldsymbol{D}$ como o produto de Kronecker entre uma matriz identidade de dimensão $N$ e um vetor de uns de dimensão $T$, a função de densidade de probabilidade de $\boldsymbol{\delta}$ dado $(\boldsymbol{\beta}, \boldsymbol{z}, \sigma)$ é:

$$
\boldsymbol{\delta} \mid \boldsymbol{\beta}, \boldsymbol{z}, \sigma \sim N_{N T}\left(\boldsymbol{\delta} \mid \boldsymbol{V} \boldsymbol{\beta}-\boldsymbol{D} \boldsymbol{z}, \sigma^{2} I_{N T}\right)
$$

Devido à natureza multivariada do problema, a equação (13) não é suficiente para definir uma densidade amostral para as observações $y_{i, t}$, pois a função de verossimilhança refere-se apenas a $\boldsymbol{\delta}$, e não aos $y_{i, t, j}$.

Para completar o modelo e usar a inferência Bayesiana, introduzem-se as $(P-1)$ dimensões restantes considerando a distribuição dos produtos dentro de cada superfície de equivalência de produção:

$$
\eta_{i, t, j}=\frac{\alpha_{j}^{q} y_{i, t, j}^{q}}{\sum_{l=1}^{P} \alpha_{l}^{q} y_{i, t, l}^{q}}
$$

sendo $\eta_{i, t}=\left(\eta_{i, t, 1}, \ldots, \eta_{i, t, P}\right)^{\prime}$ e $j=1, \ldots, P$.

Assumindo independência amostral em (14) pode-se definir a função de densidade de probabilidade:

$$
\eta_{i, t} \mid s \sim \operatorname{Dirch}_{P-1}\left(\eta_{i, t} \mid \boldsymbol{s}\right)
$$

sendo $\operatorname{Dirch}_{P-1}\left(\eta_{i, t} \mid \boldsymbol{s}\right)$ a distribuição Dirichlet com dimensão $(P-1)$ e parâmetro $\boldsymbol{s}=\left(s_{1}, \ldots, s_{P}\right)^{\prime} \in \Re_{+}^{P}$. Nota-se que $\sum_{j=1}^{P} \eta_{i, t, j}=1$ e $\eta_{i, t, j} \in(0,1)$.

Logo, a equação (13) e a equação (15) definem a função de densidade de probabilidade de $\boldsymbol{Y}$ : 


$$
\begin{aligned}
p(\boldsymbol{Y} \mid \boldsymbol{\beta}, \boldsymbol{z}, \sigma, \boldsymbol{\alpha}, q, \boldsymbol{s}, \lambda)= & N_{N T}\left(\boldsymbol{\delta} \mid \boldsymbol{V} \boldsymbol{\beta}-\boldsymbol{D} \boldsymbol{z}, \sigma^{2} I_{N T}\right) \prod_{i, t} \operatorname{Dirch}_{P-1}\left(\eta_{i, t} \mid \boldsymbol{s}\right) \\
& \prod_{i, t, j} q^{1-(1 / P)} \frac{\eta_{i, t, j}}{y_{i, t, j}}
\end{aligned}
$$

sendo $i=1, \ldots, N, t=1, \ldots, T$ e $j=1, \ldots, P$, e o último fator da equação (16) refere-se ao Jacobiano da transformação.

Portanto, define-se eficiência técnica da firma $i$, no tempo $t$ como $\exp \left(-\gamma_{i, t}\right)$, sendo $\gamma_{i, t}$ os elementos do vetor $\gamma$.

Para utilizar a abordagem Bayesiana na análise dos dados precisa-se definir as distribuições a priori para os parâmetros .

\subsubsection{Distribuição a priori para os parâmetros}

Considerando a independência entre os parâmetros, a distribuição a priori possui a seguinte estrutura:

$$
p(\boldsymbol{\beta}, \boldsymbol{z}, \sigma, \boldsymbol{\alpha}, q, \boldsymbol{s}, \lambda)=p(\boldsymbol{\beta}) p(\boldsymbol{z}) p(\sigma) p(\boldsymbol{\alpha}) p(q) p(\boldsymbol{s}) p(\lambda) .
$$

Neste trabalho considera-se as seguintes distribuições a priori para os parâmetros:

i) Distribuição a priori para $\boldsymbol{z}$ :

A distribuição de $\boldsymbol{z}$ determina a distribuição do vetor de ineficiências $\boldsymbol{\gamma}=\boldsymbol{D} \boldsymbol{z}$. Como $\boldsymbol{z} \geq 0$, a distribuição a priori escolhida é a gama:

$$
p(\boldsymbol{z} \mid \lambda) \propto \prod_{m=1}^{M} G\left(z_{m} \mid 1, \lambda\right) I_{z}(\boldsymbol{z}),
$$

sendo $\lambda$ um efeito aleatório que possui distribuição gama e $G(a, b)$ a função densidade de probabilidade da distribuição gama com média $\frac{a}{b}$ e variância $\frac{a}{b^{2}}$. 
Pode-se considerar outras especificações para a distribuição a priori do termo de ineficiência, levando em consideração características específicas de cada firma, como por exemplo, o seu tamanho ou idade, através da estrutura hierárquica (Fernández et al., 2000).

ii) Distribuição a priori para $\lambda$ :

A distribuição a priori atribuída a $\lambda$ é a gama:

$$
\lambda \sim G(\lambda \mid v, w)
$$

iii) Distribuição a priori para $\boldsymbol{\beta}$ :

A distribuição a priori para $\boldsymbol{\beta}$ é a normal $k$-variada com média $\boldsymbol{b}_{\mathbf{0}}$ e matriz de covariância $\boldsymbol{H}_{\mathbf{0}}{ }^{-1}$, truncada na região de regularidade de $\boldsymbol{\beta}$ :

$$
\boldsymbol{\beta} \sim N_{k}\left(\boldsymbol{\beta} \mid \boldsymbol{b}_{\mathbf{0}}, \boldsymbol{H}_{\mathbf{0}}^{-1}\right) I_{\beta}(\boldsymbol{\beta})
$$

Como a forma funcional a ser usada nesse trabalho é a Cobb-Douglas (eq. (7)), $I_{\beta}(\boldsymbol{\beta})$ corresponde à restrição de que os elementos de $\boldsymbol{\beta}$, exceto o intercepto, sejam maiores ou iguais a zero. O intercepto só assume valores maiores do que zero.

iv) Distribuição a priori para $\sigma$ :

A distribuição a priori de $\sigma$ é definida através da precisão $h=\sigma^{-2}$ :

$$
h \sim G\left(h \mid \frac{n_{0}}{2}, \frac{a_{0}}{2}\right) .
$$

v) Distribuição a priori para $\boldsymbol{\alpha}$ :

Como os $\alpha_{j} \in(0,1)$ e $\sum_{j=1}^{P} \alpha_{j}=1$, a distribuição a priori escolhida é a Dirichlet:

$$
\boldsymbol{\alpha} \sim \operatorname{Dirch}_{P-1}(\boldsymbol{\alpha} \mid \boldsymbol{a})
$$

sendo $\boldsymbol{a}=\left(a_{1}, \ldots, a_{P}\right)^{\prime} \in \Re_{+}^{P}$. 
vi) Distribuição a priori para $q$ :

De acordo com a teoria econômica, $q \in(1, \infty)$ determina a elasticidade de transformação, portanto, a distribuição a priori atribuída a $q$ é a gama truncada:

$$
q \sim G(q \mid 1, d) I_{(1, \infty)}(q)
$$

vii) Distribuição a priori para $s$ :

Os componentes de $\boldsymbol{s}$ assumem $P$ distribuições gama independentes:

$$
p(s)=\prod_{j=1}^{P} p\left(s_{j}\right) \propto \prod_{j=1}^{P} G\left(s_{j} \mid b_{j}, c_{j}\right),
$$

\subsubsection{Distribuição conjunta a posteriori}

A função de verossimilhança (16) junto com as distribuições a priori dos parâmetros formam a distribuição a posteriori conjunta dos dados observados e dos parâmetros:

$p(\boldsymbol{\beta}, \boldsymbol{z}, \sigma, \boldsymbol{\alpha}, q, \boldsymbol{s}, \lambda \mid \boldsymbol{Y}) \propto L(\boldsymbol{\beta}, \boldsymbol{z}, \sigma, \boldsymbol{\alpha}, q, \boldsymbol{s}, \lambda \mid \boldsymbol{Y}) p(\boldsymbol{\beta}) p(\boldsymbol{z}) p(\sigma) p(\boldsymbol{\alpha}) p(q) p(\boldsymbol{s}) p(\lambda)$

De forma equivalente, tem-se:

$$
\begin{aligned}
p(\boldsymbol{\beta}, \boldsymbol{z}, \sigma, \boldsymbol{\alpha}, q, \boldsymbol{s}, \lambda \mid \boldsymbol{Y}) \propto & N_{N T}\left(\boldsymbol{\delta} \mid \boldsymbol{V} \boldsymbol{\beta}-\boldsymbol{D} \boldsymbol{z}, \sigma^{2} I_{N T}\right) \prod_{i, t} \operatorname{Dirch}_{(P-1)}\left(\eta_{i, j} \mid \boldsymbol{s}\right) \\
& \prod_{i, t, j} q^{1-(1 / P)} \frac{\eta_{i, t, j}}{y_{i, t, j}} \prod_{m=1}^{M} G\left(z_{m} \mid 1, \lambda\right) I_{z}(\boldsymbol{z}) \\
& N_{k}\left(\boldsymbol{\beta} \mid \boldsymbol{b}_{\mathbf{0}}, \boldsymbol{H}_{\mathbf{0}}{ }^{-1}\right) I_{\beta}(\boldsymbol{\beta}) G\left(h \mid \frac{n_{0}}{2}, \frac{a_{0}}{2}\right) \operatorname{Dirch}_{P-1}(\boldsymbol{\alpha} \mid \boldsymbol{a}) \\
& G(q \mid 1, d) I_{(1, \infty)}(q) \prod_{j=1}^{P}\left[G\left(s_{j} \mid b_{j}, c_{j}\right)\right] G\left(\lambda \mid v_{0}, w_{0}\right) .
\end{aligned}
$$




\subsubsection{Distribuições condicionais completas a posteriori}

Como a distribuição a posteriori conjunta não é tratável algebricamente, a inferência é baseada em amostras obtidas através das distribuições condicionais completas a posteriori, usando algoritmos MCMC (amostrador de Gibbs para distribuições condicionais completas a posteriori com forma fechada e MetropolisHastings ou "Slice sampling" para distribuições condicionais completas a posteriori desconhecidas).

i) Distribuição condicional completa a posteriori para $\boldsymbol{z}$ :

A distribuição condicional completa a posteriori para $\boldsymbol{z}$ é uma normal $M$ variada truncada dada por:

$$
\boldsymbol{z} \mid \boldsymbol{Y}, \boldsymbol{\beta}, \sigma, \boldsymbol{\alpha}, q, \boldsymbol{s}, \lambda \sim N_{M}(\boldsymbol{m}, \boldsymbol{R}) I_{z}(\boldsymbol{z}),
$$

sendo $\boldsymbol{m}=\left(\boldsymbol{D}^{\prime} \boldsymbol{D}\right)^{-1}\left[\boldsymbol{D}^{\prime}(\boldsymbol{V} \boldsymbol{\beta}-\boldsymbol{\delta})-\sigma^{2} \lambda\right]$ e $\boldsymbol{R}=\sigma^{2}\left(\boldsymbol{D}^{\prime} \boldsymbol{D}\right)^{-1}$.

A distribuição normal $M$-variada truncada possui forma fechada, portanto pode ser amostrada através do amostrador de Gibbs. O algoritmo que descreve como amostrar dessa distribuição foi proposto por Geweke (1991).

ii) Distribuição condicional completa a posteriori para $\lambda$ :

$$
\lambda \mid \boldsymbol{Y}, \boldsymbol{\beta}, \boldsymbol{z}, \sigma, \boldsymbol{\alpha}, q, \boldsymbol{s} \sim G\left(M+v, \sum_{m=1}^{M} z_{m}+w\right) .
$$

iii) Distribuição condicional completa a posteriori para $\boldsymbol{\beta}$ :

A distribuição condicional completa a posteriori para $\boldsymbol{\beta}$ é uma normal $k$-variada truncada:

$$
\boldsymbol{\beta} \mid \boldsymbol{Y}, \boldsymbol{z}, \sigma, \boldsymbol{\alpha}, q, \boldsymbol{s}, \lambda \sim N_{k}\left(\boldsymbol{b}_{*}, \boldsymbol{H}_{*}^{-1}\right) I_{\beta}(\boldsymbol{\beta}),
$$

sendo $\boldsymbol{H}_{*}=\boldsymbol{H}_{\mathbf{0}}+\sigma^{-2} \boldsymbol{V}^{\prime} \boldsymbol{V}$ e $\boldsymbol{b}_{*}=\boldsymbol{H}_{*}^{-1}\left\{\boldsymbol{H}_{\mathbf{0}} \boldsymbol{b}_{\mathbf{0}}+\sigma^{-2} \boldsymbol{V}^{\prime}(\boldsymbol{\delta}+\boldsymbol{D} \boldsymbol{z})\right\}$. 
iv) Distribuição condicional completa a posteriori para $\sigma$ :

A distribuição condicional completa a posteriori para a precisão $h=\sigma^{-2}$ é:

$h \mid \boldsymbol{Y}, \boldsymbol{z}, \boldsymbol{\beta}, \boldsymbol{\alpha}, q, \boldsymbol{s}, \lambda \sim G\left(\frac{n_{0}+N T}{2}, \frac{a_{0}+(\boldsymbol{\delta}-\boldsymbol{V} \boldsymbol{\beta}+\boldsymbol{D} \boldsymbol{z})^{\prime}(\boldsymbol{\delta}-\boldsymbol{V} \boldsymbol{\beta}+\boldsymbol{D} \boldsymbol{z})}{2}\right)$.

v) Distribuição condicional completa a posteriori para $\boldsymbol{\alpha}$ :

A distribuição condicional completa a posteriori para $\boldsymbol{\alpha}$ é:

$$
\begin{aligned}
\boldsymbol{\alpha} \mid \boldsymbol{Y}, \boldsymbol{z}, \boldsymbol{\beta}, \sigma, q, \boldsymbol{s}, \lambda \sim & \prod_{j} \alpha_{j}^{a_{j}+s_{j} q N T-1} \prod_{i, t}\left(\sum_{j} \alpha_{j}^{q} y_{i, t, j}^{q}\right)^{-\sum_{j} s_{j}} \\
& \exp \left\{-\frac{1}{2 \sigma^{2}}(\boldsymbol{\delta}-\boldsymbol{V} \boldsymbol{\beta}+\boldsymbol{D} \boldsymbol{z})^{\prime}(\boldsymbol{\delta}-\boldsymbol{V} \boldsymbol{\beta}+\boldsymbol{D} \boldsymbol{z})\right\}
\end{aligned}
$$

sendo $i=1, \ldots, N ; t=1, \ldots, T$ e $j=1, \ldots, P$.

vi) Distribuição condicional completa a posteriori para $q$ :

A distribuição condicional completa a posteriori para $q$ possui a seguinte forma:

$$
p(q \mid \boldsymbol{Y}, \boldsymbol{z}, \boldsymbol{\beta}, \sigma, \boldsymbol{\alpha}, \boldsymbol{s}, \lambda) \propto q^{N T(P-1)} \exp (-d q) \exp \{-A(q)\} I_{(1, \infty)}(q),
$$

sendo $i=1, \ldots, N ; t=1, \ldots, T ; j=1, \ldots, P$, e

$$
A(q)=\frac{1}{2 \sigma^{2}}(\boldsymbol{\delta}-\boldsymbol{V} \boldsymbol{\beta}+\boldsymbol{D} \boldsymbol{z})^{\prime}(\boldsymbol{\delta}-\boldsymbol{V} \boldsymbol{\beta}+\boldsymbol{D} \boldsymbol{z})+\sum_{i, t, j} s_{j} \log \left(\frac{\sum_{l=1}^{P} \alpha_{l}^{q} y_{i, t, l}}{\alpha_{j}^{q} y_{i, t, j}^{q}}\right) .
$$

vii) Distribuição condicional completa a posteriori para $s$ :

Os $P$ componentes de $\boldsymbol{s}$ são gerados separadamente, e a distribuição condicional completa a posteriori para $s_{j}, j=1, \ldots, P$ possui a forma:

$$
\begin{array}{r}
p\left(s_{j} \mid \boldsymbol{Y}, \boldsymbol{z}, \boldsymbol{\beta}, \sigma, \boldsymbol{\alpha},\left\{s_{h}: h \neq j\right\}, \lambda\right) \propto \\
\frac{\Gamma\left(\sum_{l} s_{l}\right)^{N T}}{\Gamma\left(s_{j}\right)^{N T}} s_{j}^{b_{j}-1} \exp \left[-s_{j}\left\{c_{j}+\sum_{i, t} \log \left(\frac{\sum_{l} \alpha_{l}^{q} y_{i, t, l}^{q}}{\alpha_{j}^{q} y_{i, t, j}^{q}}\right)\right\}\right],
\end{array}
$$

sendo $i=1, \ldots, N ; t=1, \ldots, T$ e $j=1, \ldots, P$. 
As distribuições condicionais completas a posteriori para os parâmetros $\boldsymbol{z}, \boldsymbol{\beta}, \sigma$ e $\lambda$, possuem forma fechada, ou seja, são distribuições conhecidas (normal, gama), portanto, pode-se utilizar o amostrador de Gibbs para amostrar dessas distribuições.

As distribuições condicionais completas a posteriori para os parâmetros $\boldsymbol{\alpha}, q$ e $\boldsymbol{s}$ não possuem forma fechada e amostrar dessas distribuições não é simples. Para amostrar dessas distribuições, pode-se utilizar o algoritmo de MetropolisHastings ou o "Slice sampling".

\subsubsection{Simulação do conjunto de dados}

Para a simulação do dados são considerados $N=5$ firmas, $T=12$ tempos, $P=3$ produtos e a forma funcional Cobb-Douglas com $k=2$ variáveis regressoras com coeficientes $\boldsymbol{\beta}=0,5 \times \iota_{2}$, sendo $\iota_{2}$ um vetor de uns de dimensão 2 .

O primeiro passo para a simulação os dados é construir uma matriz $\boldsymbol{V}$ de variáveis regressoras, correspondendo ao logaritmo dos insumos. Com a forma funcional Cobb-Douglas com um único insumo $x$, a matriz $\boldsymbol{V}$ terá dimensão $(5 \times 12) \times 2$. A primeira coluna de $\boldsymbol{V}$ corresponde ao intercepto, logo, é um vetor de uns e a segunda coluna corresponde ao insumo $x$.

Para a obtenção da segunda coluna da matriz dos insumos é gerado, primeiramente, uma amostra da distribuição normal padrão de tamanho 5, representando o logaritmo do insumo de cada firma no primeiro tempo e o logarimto dos insumos nos tempos restantes é obtido por um caminho aleatório governado pela função densidade de probabilidade da distribuição normal com desvio padrão igual a 0, 01. Se valores negativos são gerados para os insumos, esses são descartados e gera-

dos novamente. A idéia desenvolvida acima foi originalmente proposta por Fernández et al. (2000).

A matriz $\boldsymbol{D}=I_{5} \otimes \iota_{12}$ especifica a eficiência constante no tempo para 
cada firma, sendo $\iota_{12}$ um vetor de uns de dimensão 12 . Os valores considerados para os parâmetros restantes são $\sigma=0,5 ; q=2 ; \boldsymbol{\alpha}=\frac{1}{3} \iota_{3} ; \boldsymbol{s}=\iota_{3}$; em que $\iota_{3}$ é um vetor de uns de dimensão 3 e $\lambda=1$, e assim obtém-se $\boldsymbol{\delta}$ (eq. (12)).

Para a obtenção da matriz dos produtos $\boldsymbol{Y}$, de dimensão $(5 \times 12) \times 3$, calcula-se $\boldsymbol{\theta}=\exp (\boldsymbol{\delta})$, assume-se que $\eta_{i, t}$ possui distribuição Dirichlet com parâmetro $\boldsymbol{s}=\iota_{3}$ e através da equação (14) obtêm-se os valores dos $P=3$ produtos para cada firma $i$ no tempo $t$.

\subsubsection{Alguns comentários sobre a implementação do programa}

O programa para obtenção de uma amostra da distribuição conjunta a posteriori (17) e, consequentemente, para as distribuições marginais de $\boldsymbol{\beta}, \boldsymbol{z}, \sigma^{2}, \boldsymbol{\alpha}, q, s$ e $\lambda$ é implementado no programa estatístico R (Venable \& Smith, 2003) e consta basicamente dos seguintes passos:

Passo 1 Defina $\boldsymbol{V}, \boldsymbol{D}, N, T, k, P, I_{N T}$.

Passo 2 Defina os hiperparâmetros:

i) $\boldsymbol{H}_{0}^{-1}, \boldsymbol{b}_{0}$ para $\boldsymbol{\beta}$;

ii) $v, w$ para $\lambda$;

ii) $n_{0}, a_{0}$ para $\sigma^{2}=\frac{1}{h}$;

iv) $\boldsymbol{a}$ para $\boldsymbol{\alpha}$

v) $d$ para $q$

vi) $b_{j}, c_{j}$ para $s_{j}$

Passo 3 Atribua valores iniciais para os parâmetros $\boldsymbol{\beta}, \boldsymbol{z}, \sigma^{2}, \boldsymbol{\alpha}, q, \boldsymbol{s}, h=\frac{1}{\sigma^{2}}$ e $\lambda$. Passo 4 Repita:

i) Calcule $\boldsymbol{\delta}$ utilizando a equação (12). 
ii) Amostre $\boldsymbol{\beta}$, eq. (20).

iii) Amostre $\boldsymbol{z}$, eq. (18).

iv) Amostre $h=\frac{1}{\sigma^{2}}$, eq. (21).

v) Amostre $\boldsymbol{\alpha}$, eq. (22).

vi) Amostre q, eq. (23).

vii) Amostre $s_{j}$, eq. (24).

viii) Amostre $\lambda$, eq. (19).

Passo 5 Repita o passo $4 M$ vezes, considere o período de aquecimento da cadeia "burn in" e o espaçamento entre os pontos amostrados "thin";

Passo 6 Calcule: $E T=\exp (-\boldsymbol{z})$, a eficiência técnica das firmas. 


\section{RESULTADOS E DISCUSSÃO}

O conjunto de dados simulado conforme descrição da seção 3.2 .6 é analisado de duas formas diferentes.

Na primeira análise, representada por MHGS, utilizam-se os algoritmos Gibbs e Metropolis-Hastings, sendo os parâmetros $h, \lambda, \boldsymbol{\beta}$ e $\boldsymbol{z}$ amostrados por Gibbs e os parâmetros $\boldsymbol{\alpha}, q$ e $\boldsymbol{s}$ amostrados por Metropolis-Hastings.

Para obter valores amostrados do parâmetro $q$ utiliza-se como função candidata a distribuição exponencial truncada no intervalo $(1, \infty)$ com parâmetro $d=1$, para o parâmetro $s$ utiliza-se a distribuição gama com parâmetro de forma $a=20$ e escala $b=20$ como função candidata, e para o parâmetro $\boldsymbol{\alpha}$ a função candidata segue o procedimento a seguir:

i) Sortear $\phi_{1}$ da distribuição normal com média zero e variância $\sigma_{1}^{2}$;

ii) sortear $\phi_{2}$ da distribuição normal com média zero e variância $\sigma_{2}^{2}$;

iii) Fazer:

$$
\phi_{1}=\log \left(\frac{a_{1}}{1-a_{1}-a_{2}}\right) \text { e } \phi_{2}=\log \left(\frac{a_{2}}{1-a_{1}-a_{2}}\right) ;
$$

iv) Encontrar $a_{1}$ e $a_{2}$;

v) Fazer $a_{3}=1-a_{1}-a_{2}$.

A segunda análise, representada por SS, difere da primeira apenas em um aspecto, os parâmetros $q$ e $\boldsymbol{s}$ são amostrados pelo algoritmo "Slice sampling", os valores iniciais e os hiperparâmetros são considerados iguais nas duas análises, sendo utilizado distribuições a priori não-informativas. 
Efetuou-se um processo de 50.000 iterações, sendo descartadas as 10.000 iniciais, para o período de aquecimento da cadeia ("burn-in"), e para assegurar a independência da amostra, considerou-se um espaçamento entre os pontos amostrados de tamanho 10 ("thin"), ou seja, obteve-se uma amostra de tamanho 10.000 para cada parâmetro.

A Tabela 4 apresenta o resumo a posteriori para cada parâmetro obtido considerando a análise MHGS e a Tabela 5 apresenta o resumo a posteriori de cada parâmetro obtido considerando a análise SS.

Tabela 4. Resumo a posteriori dos parâmetros de interesse na análise MHGS.

\begin{tabular}{ccccccc}
\hline Par. & V. verd. & Média & d.p. & $2,5 \%$ & $50 \%$ & $97,5 \%$ \\
\hline$\beta_{1}$ & 0,500 & 0,304 & 0,258 & 0,011 & 0,244 & 1,004 \\
$\beta_{2}$ & 0,500 & 0,659 & 0,590 & 0,032 & 0,503 & 2,169 \\
$h$ & 4,000 & 4,061 & 0,771 & 2,703 & 4,019 & 5,732 \\
$\alpha_{1}$ & 0,333 & 0,343 & 0,019 & 0,305 & 0,343 & 0,382 \\
$\alpha_{2}$ & 0,333 & 0,338 & 0,022 & 0,295 & 0,339 & 0,380 \\
$\alpha_{3}$ & 0,333 & 0,317 & 0,015 & 0,286 & 0,317 & 0,348 \\
$q$ & 2,000 & 1,774 & 0,225 & 1,411 & 1,744 & 2,301 \\
$\lambda$ & 1,000 & 1,007 & 0,272 & 0,529 & 0,985 & 1,606 \\
$s_{1}$ & 1,000 & 1,145 & 0,198 & 0,739 & 1,160 & 1,498 \\
$s_{2}$ & 1,000 & 1,196 & 0,211 & 0,758 & 1,208 & 1,608 \\
$s_{3}$ & 1,000 & 1,019 & 0,206 & 0,645 & 1,022 & 1,406 \\
\hline
\end{tabular}

O algoritmo Metropolis-Hastings, usado para amostrar valores de $\boldsymbol{\alpha}, q$ e $s_{j}, \quad j=1, \ldots, 3$ na análise MHGS, apresenta um bom desempenho com probabilidades de aceitação de $32,96 \%, 17,4 \%, 38,12 \%, 34,45 \%$ e 44,15\% respectivamente. $\mathrm{Na}$ análise SS, o algoritmo Metropolis-Hastings, usado para amostrar $\alpha$, também apresenta um bom desempenho com probabilidade de aceitação de 32, 96\%. 
Tabela 5. Resumo a posteriori dos parâmetros de interesse na análise SS.

\begin{tabular}{ccccccc}
\hline Par. & V. verd. & Média & d.p. & $2,5 \%$ & $50 \%$ & $97,5 \%$ \\
\hline$\beta_{1}$ & 0,500 & 0,267 & 0,250 & 0,009 & 0,195 & 0,998 \\
$\beta_{2}$ & 0,500 & 0,559 & 0,518 & 0,022 & 0,416 & 1,926 \\
$h$ & 4,000 & 4,039 & 0,791 & 2,642 & 3,986 & 5,732 \\
$\alpha_{1}$ & 0,333 & 0,343 & 0,021 & 0,301 & 0,343 & 0,386 \\
$\alpha_{2}$ & 0,333 & 0,332 & 0,023 & 0,287 & 0,332 & 0,378 \\
$\alpha_{3}$ & 0,333 & 0,323 & 0,015 & 0,294 & 0,323 & 0,355 \\
$q$ & 2,000 & 2,488 & 0,346 & 1,712 & 2,538 & 3,155 \\
$\lambda$ & 1,000 & 0,990 & 0,280 & 0,520 & 0,963 & 1,602 \\
$s_{1}$ & 1,000 & 0,775 & 0,177 & 0,377 & 0,803 & 1,055 \\
$s_{2}$ & 1,000 & 0,752 & 0,187 & 0,422 & 0,725 & 1,223 \\
$s_{3}$ & 1,000 & 0,626 & 0,266 & 0,309 & 0,565 & 1,281 \\
\hline
\end{tabular}

A análise da eficiência técnica das firmas é feita através de um posto associado as estimativas da ineficiência técnica, $z_{i}$, de cada firma $i, i=1, \ldots, 5$, a qual é dada por:

$$
E T=\exp \left(-\gamma_{(i, t)}\right)=\exp \left(-z_{i}\right),
$$

sendo $z_{i}$ a média da distribuição marginal a posteriori da ineficiência.

A Tabela 6 apresenta o resumo a posteriori do parâmetro da ineficiência obtido na primeira análise, MHGS e a Tabela 7 apresenta o resumo a posteriori do parâmetro da ineficiência obtido na segunda análise, SS.

A Figura 11 apresenta o traço e a densidade da distribuição marginal a posteriori da ineficiência $z_{i}$ específica para cada firma na análise MHGS e a Figura 13 apresenta o traço e a densidade da distribuição marginal a posteriori da ineficiência $z_{i}$ específica para cada firma na análise SS. 
Trace of $\mathbf{z 1}$

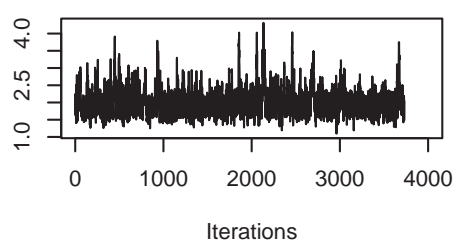

Trace of $z 2$

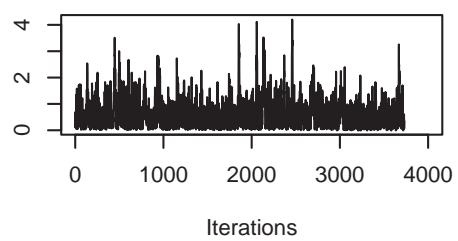

Trace of $z 3$

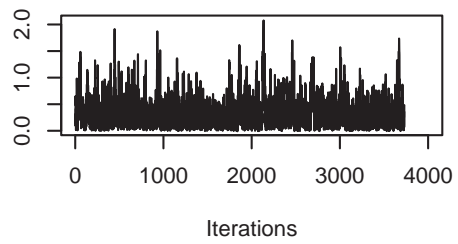

Trace of $\mathbf{z} 4$

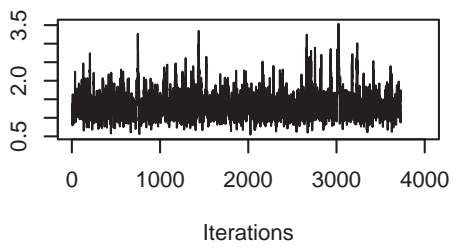

Trace of $\mathbf{z 5}$

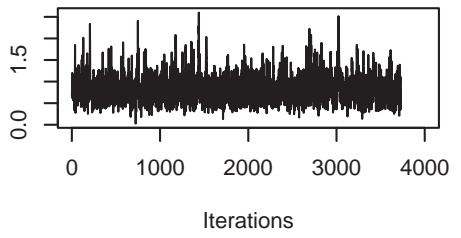

Density of $\mathbf{z 1}$

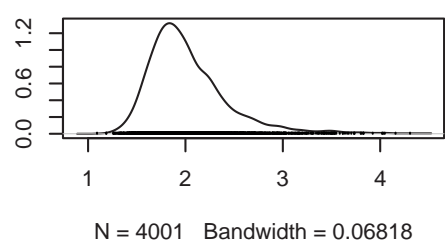

Density of z2

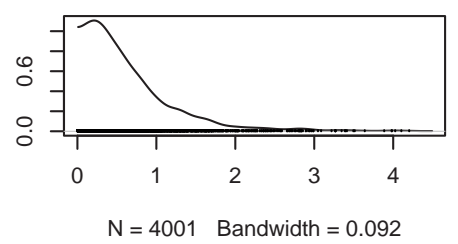

Density of z3

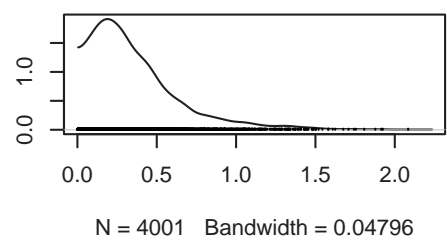

Density of $\mathbf{z 4}$

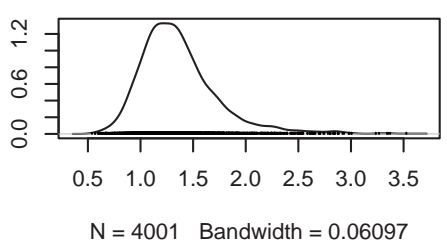

Density of z5

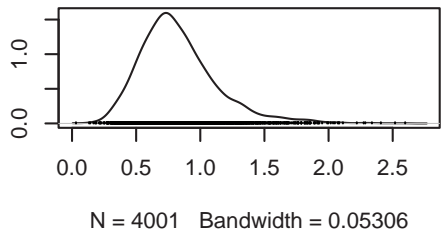

Figura 11 - Traço e densidade da distribuição marginal a posteriori do parâmetro $z$ na análise MHGS. 

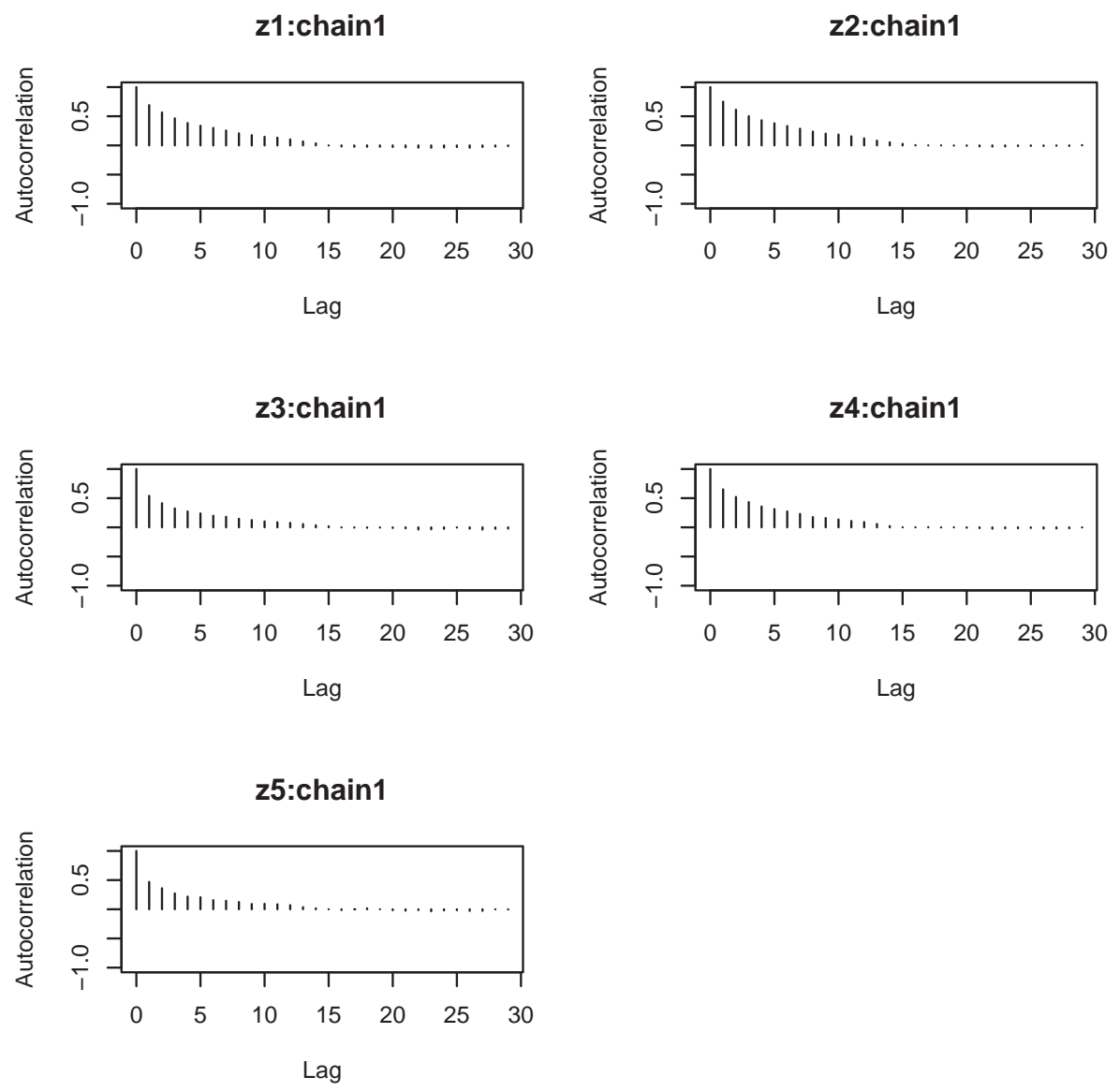

Figura 12 - Autocorrelação da amostra gerada para o parâmetro $z=\left(z_{1}, \ldots, z_{5}\right)$ na análise MHGS. 
Trace of $z 1$

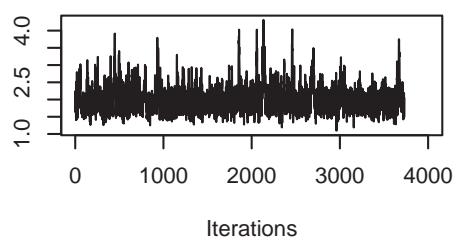

Trace of $z 2$

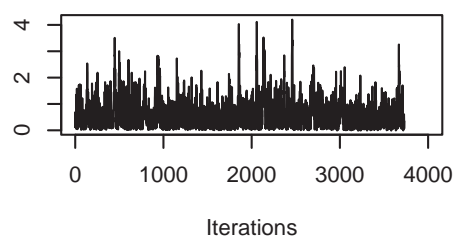

Trace of $z 3$

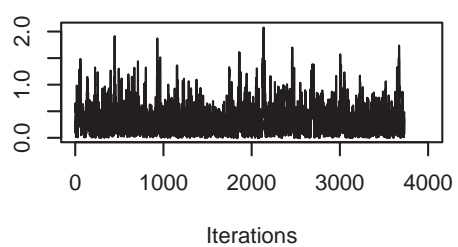

Trace of $\mathbf{z 4}$

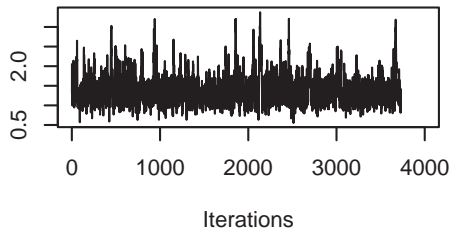

Trace of $\mathbf{z 5}$

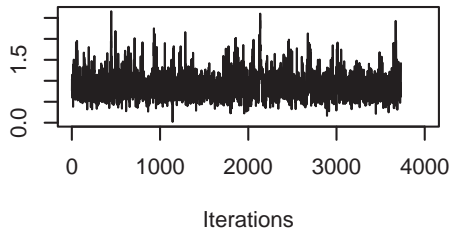

Density of z1

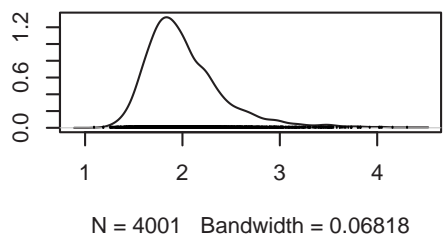

Density of z2

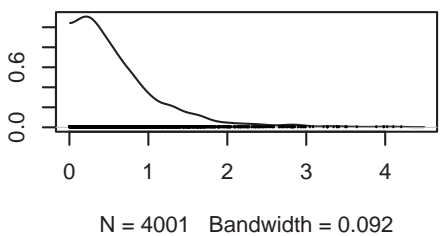

Density of z3

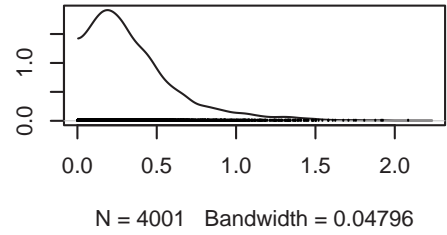

Density of $\mathbf{z 4}$

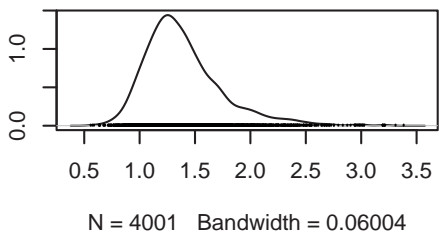

Density of $\mathbf{z 5}$

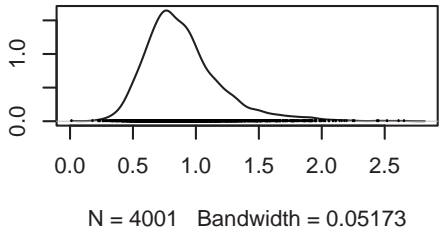

Figura 13 - Traço e densidade da distribuição marginal a posteriori do parâmetro z na análise SS. 

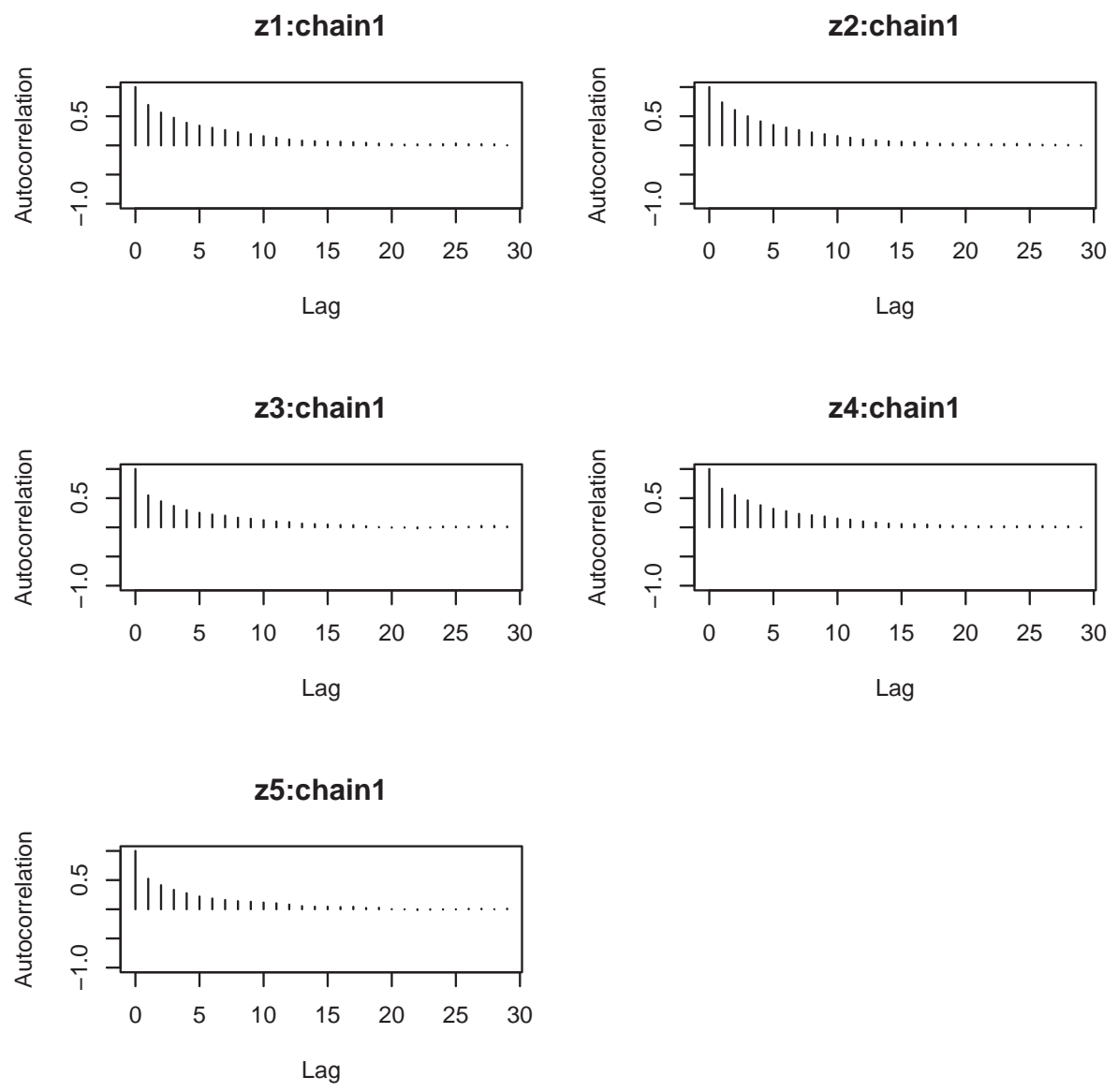

Figura 14 - Autocorrelação da amostra gerada para o parâmetro $z=\left(z_{1}, \ldots, z_{5}\right)$ na análise SS. 
As Figuras 12 e 14 apresentam os gráficos da autocorrelação para parâmetro da ineficiência $z$ nas análises MHGS e SS respectivamente, e conclui-se que não existe evidência de ineficiência na simulação.

A convergência das cadeias de todos os parâmetros do modelo foram monitoradas através da visualização gráfica do traço, densidade e dos critérios disponíveis no pacote CODA do programa R, não existindo evidências contra a convergência.

Tabela 6. Resumo a posteriori do vetor de ineficiências na análise MHGS.

\begin{tabular}{ccccccc}
\hline Par. & V. Verd. & Média & d.p. & $2,5 \%$ & $50 \%$ & $97,5 \%$ \\
\hline$z_{1}$ & 2,015 & 1,983 & 0,408 & 1,403 & 1,906 & 3,033 \\
$z_{2}$ & 0,787 & 0,635 & 0,596 & 0,026 & 0,472 & 2,173 \\
$z_{3}$ & 0,286 & 0,312 & 0,269 & 0,015 & 0,241 & 1,032 \\
$z_{4}$ & 1,536 & 1,357 & 0,367 & 0,825 & 1,301 & 2,298 \\
$z_{5}$ & 1,257 & 0,825 & 0,303 & 0,358 & 0,778 & 1,592 \\
\hline
\end{tabular}

Tabela 7. Resumo a posteriori do vetor de ineficiências na análise SS.

\begin{tabular}{ccccccc}
\hline Par. & V. Verd. & Média & d.p. & $2,5 \%$ & $50 \%$ & $97,5 \%$ \\
\hline$z_{1}$ & 2,015 & 2,013 & 0,397 & 1,459 & 1,932 & 3,024 \\
$z_{2}$ & 0,787 & 0,625 & 0,563 & 0,026 & 0,474 & 2,159 \\
$z_{3}$ & 0,286 & 0,355 & 0,284 & 0,020 & 0,286 & 1,132 \\
$z_{4}$ & 1,536 & 1,396 & 0,365 & 0,880 & 1,325 & 2,346 \\
$z_{5}$ & 1,257 & 0,893 & 0,303 & 0,449 & 0,844 & 1,670 \\
\hline
\end{tabular}

A Tabela 8 mostra a classificação da eficiência técnica da firma mais eficiente para a menos eficiente nas duas análises.

O índice de eficiência técnica das firmas analisadas nesse trabalho manteve sua classificação nas duas análises, independente do algoritmo utilizado para 
amostrar as distribuições dos parâmetros.

Tabela 8. Índice de Eficiência Técnica

\begin{tabular}{cccc}
\hline Firma. & V. Verd. & $E T_{M H G S}$ & $E T_{S S}$ \\
\hline$F_{3}$ & 0,751 & 0,732 & 0,701 \\
$F_{2}$ & 0,455 & 0,530 & 0,535 \\
$F_{5}$ & 0,285 & 0,438 & 0,409 \\
$F_{4}$ & 0,215 & 0,257 & 0,248 \\
$F_{1}$ & 0,133 & 0,138 & 0,134 \\
\hline
\end{tabular}

Segundo os resultados apresentados neste trabalho, pode-se concluir que os algoritmos Metropolis-Hastings e o "Slice sampling" obtiveram resultados semelhantes relativos aos resumos a posteriori dos parâmetros de interesse obtidos nas análises MHGS e SS.

O método "Slice sampling" requer um menor esforço na implementação do algoritmo, mas neste caso, apresentou maior tempo computacional para a execução do programa. O método "Slice sampling" demorou 5 horas e 17 minutos para amostrar 50.000 pontos e o método Metropolis-Hastings demorou 2 horas e 10 minutos para amostrar a mesma quantidade pontos.

Possíveis extensões deste trabalho são: a análise de conjuntos de dados reais, que foram cedidos pelos pesquisadores Oscar Tupy e Geraldo da Silva e Souza da Empresa Brasileira de Pesquisa Agropecuária (EMBRAPA); a inserção de covariáveis no modelo, com a finalidade de analisar a eficiência técnica das firmas variando no tempo; a utilização de outras formas funcionais, tais como a Translog, para estimar a fronteira de produção; a utilização do método "Slice sampling" multivariado e implementação do algoritmo no programa WinBugs. 


\section{CONCLUSÕES}

\section{Pode-se concluir que:}

i) das 5 firmas analisadas, a firma 3 foi a mais eficiente e a firma 1 a menos eficiente;

ii) o algoritmo utilizado para amostrar distribuições com forma desconhecida que obteve melhor desempenho foi o Metropolis-Hastings.

A proposta original deste trabalho foi cumprida, mostrando como calcular eficiência técnica de empresas que possuem múltiplos produtos, usando a abordagem Bayesiana. 


\section{REFERÊNCIAS BIBLIOGRÁFICAS}

ADAMS, R.; BERGER, A.; SICKLES, R. Semiparametric approaches to stochastics panel frontiers with applications in the banking industry. Journal of Business and Economic Statistics, v.17, p.349-358, 1999.

AIGNER, D.J.; CHU, S.F. On estimating the industry production functions. American Economic Review, v.58, n.3, p.826-839, 1968.

AIGNER, D.J.; LOVELL, C.A.K.; SCHMIDT, P. Formulation and estimation of stochastic frontier production function models. Journal of Econometrics, v.6, p.21-37, 1977.

ALI, A.I; SEIFORD, L.M. The mathematical programming approach to efficiency analysis. In: FRIED, H.O.; LOVELL, C.A.K.; SCHMIDT, S.S. (Ed.). The measurement of productive efficiency: techniques and applications. New-York: Oxford University Press, 1993. p.120-159.

BAYES, T. An essay towards solving a problem in the doctrine of chances. Philosophical Transactions of the Royal Society of London, v.53, p.370-418, 1763.

BOX, G.E.P.; TIAO, G.C. Bayesian inference in statistical analysis. New York: Wiley, 1992. 588p.

CARLIN, B.P.; LOUIS, T.A. Bayes and empirical Bayes methods for data analysis. London: Chapman \& Hall, 1996. 399p. 
CASELlA, G.; GEORGE, E.I. Explaining the Gibbs sampler. The American Statistician, v.46, n.3, p.167-174, 1992.

CHARNES, A.; COOPER, W.W.; LEWIN, A.Y.; SEIFORD, L.M. Data envelopment analysis: theory, methodology and applications. Boston: Klumber Academic Publishers, 1995. 352p.

CHIB, S.; GREENBERG, E. Understanding the Metropolis-Hastings algorithm. The American Statistician, v.49, n.4, p.327-335, 1995.

COELLI, T.; RAO, D.S.P.; BATTESE, G.E. An introduction to efficiency and productivity analysis. Boston: Klumber Academic Publishers, 1999. 275p.

CUESTA, R.A.; O’DONNELL, C.J.; COELLI, T.J.; SINGH, S. Imposing curvature conditions on a production frontier: with application to Indian dairy processing plants. http://www.une.edu.au/febl/EconStud/emet/cepa2_01.PDF (31 Mar. 2003)

FERNÁNDEZ, C.; KOOP, G.; STEEL, M.F.J. A Bayesian analysis of multipleoutput production frontiers. Journal of Econometrics, v.98, p.47-79, 2000.

FERNÁNDEZ, C.; OSIEWALSKI, J.; STEEL, M.F.J. On the use of panel data in stochastic frontier models with improper priors. Journal of Econometrics. v.79, p.169-193, 1997.

GAMERMAN, D. Markov chain Monte Carlo: stochastic simulation for Bayesian inference. London: Chapman and Hall, 1997. 245p.

GELFAND, A.E. Gibbs sampling. Journal of the American Statistical Association, v.585, p.1300-1304, 2000.

GELFAND, A.E.; HILLS, S.E.; RACINE-POON, A.; SMITH, A.F.M. Illustration of Bayesian inference in normal data models using Gibbs sampling. Journal of the American Statistical Association, v.85, p.972-985, 1990. 
GELMAN, A.; CARLIN, J.B.; STER, H.S.; RUBIN, D.B. Bayesian data analysis. London: Chapman and Hall, 2003. 668p.

GEMAN, S.; GEMAN, D. Stochastic relaxation, Gibbs distributions, and the Bayesian restoration of images. IEEE Transactions on Pattern Analysis and Machine Intelligence, v.6, p.721-741, 1984.

GEWEKE, J. Efficient simulation from the multivariate normal and Student-t distributions subject to linear constraints and the evaluation of constraint probabilities. http://www.biz.uiowa.edu/faculty/jgeweke/papers/paper47/paper47.pdf Mai. 2003)

GILKS, W.R.; RICHARDSON, S.; SPIEGELHALTER, D.J. Markov chain Monte Carlo in practice. London: Chapman and Hall, 1997. 486p.

GREENE, W.H. A gamma-distributed stochastic frontier model. Journal of Econometrics, v.46, p.141-164, 1990.

GUJARATI, D.N. Basic econometrics. Singapore: McGraw-Hill International Editions, 1995. 838p.

HASTINGS, W.K. Monte Carlo sampling methodos using Markov chains and their applications. Biometrika, v.57, p.97-109, 1970.

JEFFREYS, H. Theory of probability, 3. ed. Oxford: Clarendon Press, 1961. $447 \mathrm{p}$.

KOOP, G.; STEEL, M.F.J. Bayesian analysis of stochastic frontier models. In: BALTAGI, B. (Ed.). A companion to theoretical econometrics. Oxford: Blackwell, 2001. p.520-573. 
KOOP, G.; STEEL, M.F.J.; OSIEWALSKI, J. Posterior analysis of stochastic frontier models using Gibbs sampling. Computational Statistics, v.10, p.353-373, 1995.

KOOP, G.; OSIEWALSKI, J.; STEEL, M.F.J. Bayesian efficiency analysis through individual effects: hospital cost frontiers. Journal of Econometrics, v.76, p.77$105,1997$.

KUMBHAKAR, S. The specification of technical and allocative inefficiency of multiple product firms in stochastic production and profit frontiers. Journal of Quantitative Economics, v.3, p.213-223, 1987.

LEANDRO, R.A. Introdução à estatística Bayesiana. Piracicaba: Escola Superior de Agricultura Luiz de Queiroz, Universidade de São Paulo, Departamento de Ciências Exatas, 2001. 51p.

LOTHGREN, M. Generalized stochastic frontier production models. Economics Letters, v.57, p.255-259, 1997.

LOVELL, C.A.K. Production frontiers and productive efficiency. In: FRIED, H.O.; LOVELL, C.A.K.; SCHMIDT, S.S. (Ed.). The measurement of productive efficiency: techniques and applications. New-York: Oxford University Press, 1993. p.3-67.

LOVELL, C.A.K. Linear programming approaches to the measurement and analysis of productive efficiency. Top, v.2, p.175-248, 1994.

MEDRANO, L.A.T. Análise Bayesiana de modelos de fronteiras de produção estocásticas. Rio de Janeiro, 2003. 107p. Dissertação (M.S.) - Universidade Federal do Rio de Janeiro. 
MEEUSEN, W.; VAN DEN BROECK, J. Efficiency estimation from Cobb-Douglas production functions with composed error. International Economic Review, v.18, p.435-444, 1977.

METROPOLIS, N.; ROSEnBLUTH, A.W., ROSENBLUTH, M.N.; TELLER, A.H.; TELLER, E. Equations of state calculations by fast computing machines. Journal of Chemical Physics, v.21, p.1087-1091, 1953.

NEAL, R.M. Slice Sampling. The Annals of Statistics, v.31, n.3, p.705-767, 2003.

NICHOLSON, W. Microeconomic theory: basic principles and extensions. New York: Thomson Learning, 2002. 748p.

OSIEWALSKI, J.; STEEL, M.F.J. Numerical tools for the Bayesian analysis of frontier models. Journal of Productivity Analysis, v.10, p.103-117, 1998.

PEREIRA FILHO, C. A. Eficiência econômica da pequena produção familiar agrícola no Recôncavo do Estado da Bahia: uma análise não-paramétrica de fronteiras de produção multi-produto. Piracicaba, 2000. 122p. Tese (Doutorado) - Escola Superior de Agricultura "Luiz de Queiroz", Universidade de São Paulo.

PINILLA, A.A. La medición de la eficiencia y la productividad. Madrid: Ediciones Pirámide, 2001. 363p.

POWELL, A.; GRUEN, F. The constant elasticity of transformation production frontier and linear supply system. International Economic Review, v.9, p.315$328,1968$.

SEIFORD, L.M. Data envelopment analysis: the evolution of de state of the art (1978-1995). Journal of Productivity Analysis, v.7, p.99-138, 1996.

SEIFORD, L.M.; THRALL, R.M. Recent developments in DEA: the mathematical approach to frontier analysis. Journal of Econometrics, v.46, p.7-38, 1990. 
SOUZA, G.S. Funções de produção: uma abordagem estatística com o uso de modelos de encapsulamento de dados. http://www.embrapa.br/unidades/uc/sge/texto17.pdf (20 Set. 2003)

STEVENSON, R.E. Likelihood functions for generalised stochastic frontier estimation. Journal of Econometrics, v.13, p.57-66, 1980.

TSIONAS, E.G. Full likelihood inference in normal-gamma stochastic models. Journal of Productivity Analysis, v.13, p.183-205, 2000.

TSIONAS, E.G. Stochastic frontier models with random coefficients. Journal of Applied Econometrics, v.17, p.127-147, 2002.

THOMPSON, A.A.; FORMBY, J.P. Microeconomia da firma: teoria e prática. Rio de Janeiro: Prentice-Hall do Brasil, 1998. 358p.

VAN DEN BROECK, J.; KOOP, G.; OSIEWALSKI, J.; STEEL, M.F.J. Stochastic frontier models: a Bayesian perspective. Journal of Econometrics, v.61, p.273303, 1994.

VENABLE, W.N.; SMITH, D.M. An Introduction to R. Notes on R: a programming environment for data analysis and graphics. Version 1.7.0 (16 Abr. 2003) 